\title{
Low-temperature properties of the Hubbard model on highly frustrated one-dimensional lattices
}

\author{
O. Derzhko, ${ }^{1,2,3}$ J. Richter, ${ }^{3}$ A. Honecker, ${ }^{4}$ M. Maksymenko, ${ }^{1}$ and R. Moessner ${ }^{2}$ \\ ${ }^{1}$ Institute for Condensed Matter Physics, National Academy of \\ Sciences of Ukraine, 1 Svientsitskii Street, L'viv-11, 79011, Ukraine \\ ${ }^{2}$ Max-Planck-Institut für Physik komplexer Systeme, Nöthnitzer Straße 38, 01187 Dresden, Germany \\ ${ }^{3}$ Institut für theoretische Physik, Universität Magdeburg, P.O. Box 4120, 39016 Magdeburg, Germany \\ ${ }^{4}$ Institut für Theoretische Physik, Georg-August-Universität Göttingen, \\ Friedrich-Hund-Platz 1, 37077 Göttingen, Germany
}

(Dated: November 2, 2018)

\begin{abstract}
We consider the repulsive Hubbard model on three highly frustrated one-dimensional lattices sawtooth chain and two kagomé chains - with completely dispersionless (flat) lowest single-electron bands. We construct the complete manifold of exact many-electron ground states at low electron fillings and calculate the degeneracy of these states. As a result, we obtain closed-form expressions for low-temperature thermodynamic quantities around a particular value of the chemical potential $\mu_{0}$. We discuss specific features of thermodynamic quantities of these ground-state ensembles such as residual entropy, an extra low-temperature peak in the specific heat, and the existence of ferromagnetism and paramagnetism. We confirm our analytical results by comparison with exact diagonalization data for finite systems.
\end{abstract}

PACS numbers: 71.10.-w, 71.10.Fd

Keywords: Hubbard model, sawtooth chain, kagomé chains, flat bands, ferromagnetism

\section{INTRODUCTION AND MOTIVATION}

The Hubbard model is a particularly simple model for strongly interacting electrons in solids $\stackrel{1}{*}$ Nevertheless, rigorous analysis of the model is a difficult task and exact statements about its properties are notoriously rare $\stackrel{1,2,3}{ } \mathrm{~A}$ number of rigorous/exact results have been obtained for the Hubbard model on some specific lattices, see, e.g., Refs. 4, 5, 6, 7, 8, 9, 10, 11, 12. In particular, in the context of the origin of ferromagnetism in itinerant electron systems $\underline{13}$ different lattices supporting dispersionless (flat) single-electron band were studied in some detail $, 4,5,6,14,15$ In the last years the theory of flat-band ferromagnetism has been developed further $16,17,18,19,20,21,22$ Although at first glance one may think that the lattices admitting rigorous treatment are rather artificial, nowadays new possibilities to design interacting lattice system with controlled geometry emerge. Thus, modern strategies in chemistry open a route to synthesize new materials with a desired lattice structure and intersite interaction $\stackrel{23}{\underline{n}}$ Furthermore, recent progress in nanotechnology allows the fabrication of quantum dot superlattices and quantum wire systems with any type of lattice $\stackrel{20}{=}$ Another rapidly developing field is the controlled setup of optical lattices for cold atoms. $\underline{10,24,25}$

On the other hand, during the past years it has been noticed that exact ground states of the quantum $X X Z$ Heisenberg antiferromagnet can be constructed at high magnetic fields for a large class of geometrically frustrated lattices ${ }^{26}$ These states, called independent or isolated localized magnons, are localized on nonoverlapping restricted areas of the lattice and they clearly manifest themselves in various peculiarities of the low-temperature strong-field properties of the spin systems (macroscopic magnetization jump, $\stackrel{26}{26}$ field-

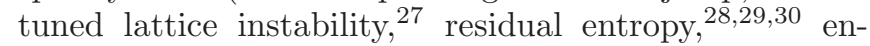
hanced magnetocaloric effect, $\underline{28,31}$ order-disorder phase transition below the saturation field ${ }^{29,30}$ etc.). Interestingly, flat-band ferromagnetism of Hubbard electrons exhibits some similarities to the localized-magnon effect for $X X Z$ Heisenberg antiferromagnets on certain frustrated lattices $\frac{11,12,32}{11}$ Note, however, that while the one-particle description may be identical, the manyparticle picture is obviously different. While the spin model can be viewed as a hard-core bosonic system with nearest-neighbor intersite repulsion, the electronic Hubbard model is a two-component fermionic system with on-site repulsion between different species. Nevertheless, it has been found recently $11,12,32$ that several ideas developed for the Heisenberg model can be carried over to the Hubbard model.

In the present paper we consider the repulsive Hubbard model on a class of one-dimensional frustrated lattices, namely the sawtooth chain and two different kagomé chains (see Figs. 19, 10, and 1 c). These Hubbard systems have highly degenerate ground states for certain electron numbers $n \leq n_{\max }, n_{\max } \propto N$, where $N$ is the number of lattice sites. We will give explicit analytical expressions for all these ground states. Their number grows exponentially with the system size and can be counted by a mapping of the electronic problem onto a one-dimensional classical hard-dimer gas. Moreover, these systems show saturated ground-state ferromagnetism for particular values of electron number $n$, i.e., the square of the total spin is $\boldsymbol{S}^{2}=(n / 2)(n / 2+1)$. Although the sawtooth chain on the one hand and the kagomé chains on the other hand belong to different types of flat-band ferromagnets, the Hubbard model on all three lattices exhibits 
(a)
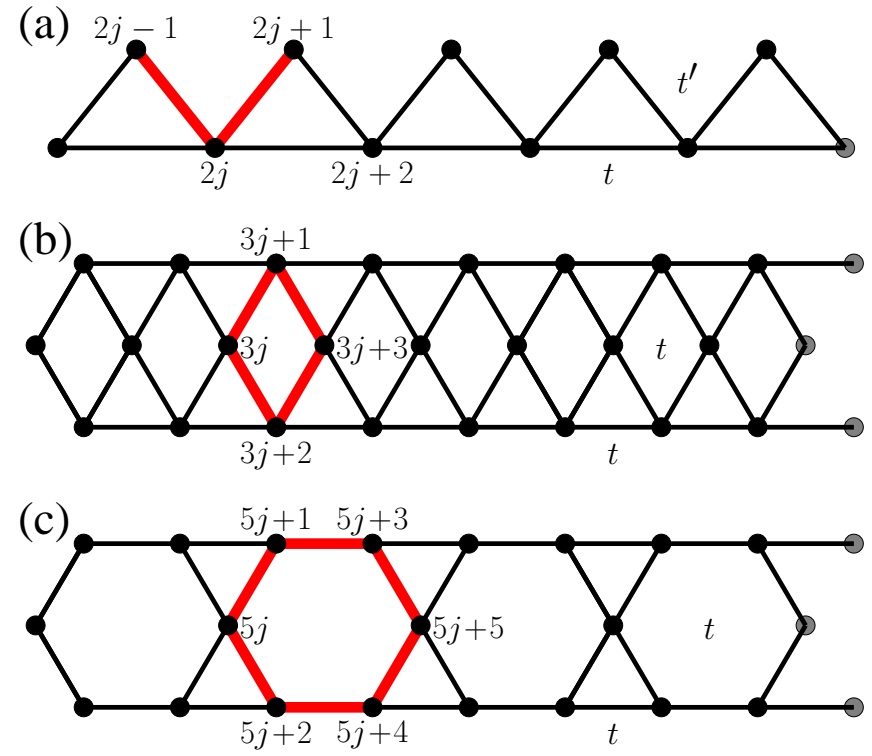

FIG. 1: (Color online) Three one-dimensional lattices considered in this paper: (a) the sawtooth chain, (b) the kagomé chain I, and (c) the kagomé chain II. For the sawtooth Hubbard chain the hopping parameter along the zig-zag path $t^{\prime}$ is $\sqrt{2}$ times larger than the hopping parameter $t>0$ along the base line. For the kagomé Hubbard chains all the hopping parameters $t>0$ are identical. Bold (red) lines denote the minimal trapping cells for localized electrons.

an identical thermodynamic behavior at low temperatures around a certain value of the chemical potential $\mu_{0}$ if $N \rightarrow \infty$. Indeed, while the sawtooth lattice being a one-dimensional version of Tasaki's model is an example of the "cell construction" $, 5,6$ the kagomé chain I and the kagomé chain II belong to Mielke's class of "line graphs", where the kagomé chain I is the line graph of the two-leg ladder 33,34 and the kagomé chain II is the line graph of a decorated two-leg ladder. The number of sites in the unit cell is 2,3 , and 5 for the sawtooth chain, kagomé chain I, and kagomé chain II, respectively. More differences can be seen in the single-electron energies for these lattices: although the tight-binding model of all three lattices exhibits a dispersionless (flat) lowest-energy band, the next dispersive band is separated by a finite gap for the sawtooth chain, but it touches the flat band at one point in momentum space in the case of the kagomé chains (for more details see Sec. III). We mention that all of the three lattices were discussed previously in the context of various problems of strongly correlated systems, see, e.g., Refs. 35, 36, 37, 38, 39, $40,41,42,43,44,45,46$.
We consider the standard Hubbard Hamiltonian

$$
\begin{aligned}
H & =\sum_{\sigma=\uparrow, \downarrow} H_{0 \sigma}+H_{\mathrm{int}}+\mu \sum_{i}\left(n_{i, \uparrow}+n_{i, \downarrow}\right), \\
H_{0 \sigma} & =\sum_{\langle i, j\rangle} t_{i, j}\left(c_{i, \sigma}^{\dagger} c_{j, \sigma}+c_{j, \sigma}^{\dagger} c_{i, \sigma}\right), \\
H_{\mathrm{int}} & =U \sum_{i} n_{i, \uparrow} n_{i, \downarrow} .
\end{aligned}
$$

The sums run over $N$ lattice sites $i$ or over the nearestneighbor pairs $\langle i, j\rangle$, and periodic boundary conditions are imposed, $c_{i, \sigma}^{\dagger}\left(c_{i, \sigma}\right)$ are the usual fermion creation (annihilation) operators. For the sawtooth chain the hopping parameters along the zig-zag path $t^{\prime}$ are $\sqrt{2}$ times larger than the ones along the base line $t>0$ (then the lowest single-electron band is completely flat, see Sec. III). For the kagomé chains all the hopping parameters equal $t>0$ (then the lowest single-electron band is completely flat, see Sec. IIII). $U \geq 0$ is the onsite Coulomb repulsion for electrons with different spins. With further statistical-mechanics calculation in mind we also introduce the term with chemical potential $\mu$. Note that the sign of the term with $\mu$ in Eq. (1.1) is chosen to have direct correspondence between the chemical potential $\mu$ for the electronic model and the magnetic field $h$ for the respective antiferromagnetic spin model $\underline{11,12,32}$ In what follows we often will set $t=1$.

The remainder of the paper is organized as follows. We begin with a brief summary of our main results, Sec. [II. In Sec. III we discuss the tight-binding model of noninteracting electrons [we set $U=0$ in Eq. (1.1)] for the three chains. In Sec. IV we construct the complete set of exact many-electron ground states of the repulsive Hubbard model on the three lattices for the electron numbers $n \leq n_{\max }$, where $n_{\max }=\mathcal{N}$ or $\mathcal{N}+1$ and $\mathcal{N} \propto N$, and discuss some properties of these states. Moreover, we explain the mapping of a certain subset of these states onto spatial configurations of classical hard dimers on a simple chain. This mapping is crucial to calculate the exact degeneracies of the ground states for electron numbers $n \leq \mathcal{N}$ and to determine the residual entropy caused by these states. In Sec. $\nabla$ we calculate analytically the contribution of the highly degenerate groundstate manifold to the grand-canonical partition function of the Hubbard model on the respective one-dimensional lattices. This contribution dominates at low temperatures when the chemical potential $\mu$ is around a certain value $\mu_{0}\left(\mu_{0}=2 t\right.$ for all three lattices). We also calculate analytically the low-temperature behavior of several thermodynamic quantities such as the average number of electrons, the entropy, and the specific heat, and we compare these analytical findings with numerical results obtained by exact diagonalization for finite lattices. Moreover, we use exact diagonalization for finite lattices to discuss the influence of small deviations from the ideal geometry (leading to a dispersion of the former flat band) on the low-temperature thermodynamics. In Sec. VI we use the analytical findings based on the localized-state 
picture and complementary numerical data from exact diagonalization of finite systems for the discussion of the ground-state magnetic properties of the considered Hubbard chains. In particular, we discuss the appearance of ferromagnetism and paramagnetism. In Sec. VII we briefly discuss the relation between the electron models and corresponding localized-spin models. Finally we summarize our findings in Sec. VIII Some auxiliary calculations are collected in Appendices.

\section{SUMMARY OF RESULTS}

Compared to previous work on flat-band Heisenberg $X X Z$ magnets, the theme of this paper is the new physics which arises in itinerant magnets in which (a) the mobile degrees of freedom are subject to the Pauli principle and (b) the Hamiltonian exhibits full SU(2) symmetry; in particular, these features set this work apart from previous work on $X X Z$ models.

Previous studies on itinerant flat-band ferromagnets were focused on particular values $n_{\mathrm{f}}$ of the electron number $n$ for which ground states with saturated ferromagnetism exist. By contrast, we characterize the complete set of ground states at electron numbers $n \leq n_{\mathrm{f}}$, count their numbers, and use them to obtain explicitly the lowtemperature thermodynamic quantities as well as the average ground-state magnetic moments.

Our results cover, on an equal footing, two families of flat-band ferromagnets in one dimension. The first are those obtained from Tasaki's cell construction and those described by Mielke's line-graph construction.

Somewhat unusually for a strongly interacting itinerant many-body system, for these lattices we provide an explicit construction of the full set of exact ground states, for a finite range of doping. The construction of the ground states is based on a mapping to hard-core dimers on an appropriate one-dimensional structure.

This result enables us to obtain the corresponding partition functions, and hence the low-temperature thermodynamics, of the magnets in the low-doping regime.

The most salient consequences concern (i) the entropy, (ii) the low-temperature specific heat, (iii) the dependence of the average number of electrons on the chemical potential and (iv) the magnetic properties.

Regarding (i) the flat band in the one-particle energies leads to a huge degeneracy of the many-body ground states for a certain range of electron densities resulting in a residual entropy in the thermodynamic limit. This highly degenerate ground-state manifold has a great impact on the low-temperature physics in the low-doping regime. In particular, an extra low-temperature peak in the specific heat appears which is related to an emerging low-energy scale separated from the energy scale determined by the value of the hopping integral. Moreover, the zero-temperature average number of electrons exhibits a jump at a certain value of the chemical potential. Regarding (iv) the structure impressed on the many-body wave-function by the Pauli principle leads to a degree of ferromagnetism in finite systems which varies with electron number $n$; there are specific fillings at which ferromagnetism is saturated [the square of the total spin is $\left.\boldsymbol{S}^{2}=(n / 2)(n / 2+1)\right]$ while at others it is only partially developed. However, in the thermodynamic limit the region of electron density $n / N$, for which ground-state ferromagnetism exists shrinks to one point $(n / N=1 / 2$ for the sawtooth chain, $n / N=1 / 3$ for the kagomé chain I, and $n / N=1 / 5$ for the kagomé chain II). For lower electron densities the ground state is paramagnetic and the low-temperature behavior of the zero-field susceptibility follows a Curie law.

Finally we emphasize here that the localized-magnon states for the $X X Z$ Heisenberg antiferromagnet on all three lattices can be also mapped onto a one-dimensional model of hard dimers. Interestingly, due to the Pauli principle, the localized-electron states are less constraining than the respective localized-magnon states. As a result the manifold of localized states for the electronic system is much larger than that for the magnon system.

\section{NON-INTERACTING ELECTRONS. TRAPPED ELECTRON STATES}

For zero Coulomb interaction $U$ the diagonalization of the Hamiltonian (1.1) is straightforward. Nevertheless, we start with the discussion of this case, since it provides some important results which are relevant for the case $U>0$, too. The sawtooth chain has been studied in great detail before (see, e.g., Ref. 32). We will therefore just recall the main results for this case and give further details only for the two kagomé chains. For brevity, we may also omit spin indices as irrelevant in this section.

\section{A. Sawtooth chain}

The sawtooth chain consists of $\mathcal{N}=N / 2$ cells, each cell contains two sites, see Fig. 17. Hence, there are two branches of single-particle energies which read 32

$$
\varepsilon_{1,2}(\kappa)=t \cos \kappa \mp \sqrt{t^{2} \cos ^{2} \kappa+2 t^{\prime 2}(1+\cos \kappa)}+\mu .
$$

For $t^{\prime}=\sqrt{2} t>0$ the lowest single-electron band becomes flat, $\varepsilon_{1}(\kappa)=\varepsilon_{1}=-2 t+\mu$ and one can write down creation operators for a corresponding set of eigenstates which are localized in a valley with index $j: 32$

$$
l_{2 j}^{\dagger}=c_{2 j-1}^{\dagger}-\sqrt{2} c_{2 j}^{\dagger}+c_{2 j+1}^{\dagger} .
$$

This set of localized single-electron states is a convenient starting point for the construction of the many-electron ground states of the Hamiltonian (1.1) in the subspaces with electron numbers $n=2, \ldots, \mathcal{N}$. Moreover, this "localized" point of view allows a useful simple geometrical interpretation, see below. 


\section{B. Kagomé chain I}

Consider next the kagomé chain I, see Fig. 1b. It consists of $\mathcal{N}=N / 3$ cells, each cell contains three sites. After standard transformations we get the diagonal form of the Hamiltonian

$$
\begin{aligned}
H_{0}+\mu \sum_{i} n_{i} & =\sum_{p=1}^{3} \sum_{\kappa} \varepsilon_{p}(\kappa) \alpha_{p, \kappa}^{\dagger} \alpha_{p, \kappa} \\
\varepsilon_{1}(\kappa) & =-2 t+\mu \\
\varepsilon_{2}(\kappa) & =2 t \cos \kappa+\mu \\
\varepsilon_{3}(\kappa) & =2 t(1+\cos \kappa)+\mu
\end{aligned}
$$

with $\kappa=2 \pi m / \mathcal{N}, m \in \mathbb{Z},-\mathcal{N} / 2<m \leq \mathcal{N} / 2$. The lowest energy band is flat, $\varepsilon_{1}(\kappa)=\varepsilon_{1}=-2 t+\mu$. Note, however, that a state with $\kappa=\pi$ (it does exist if $\mathcal{N}$ is even) from the dispersive band $\varepsilon_{2}(\kappa)$ has also the energy $\varepsilon_{1}=-2 t+\mu$, i.e., the next band touches the lowest flat band at $\kappa=\pi$. The $\kappa$-dependent single-electron states are given by $\alpha_{p, \kappa}^{\dagger}|0\rangle, p=1,2,3$, with

$$
\begin{aligned}
\alpha_{1, \kappa}^{\dagger} & =-\frac{1}{\sqrt{2 \mathcal{N}(2+\cos \kappa)}} \sum_{j=0}^{\mathcal{N}-1} e^{-i \kappa j} l_{3 j}^{\dagger}, \\
l_{3 j}^{\dagger} & =c_{3 j}^{\dagger}-c_{3 j+1}^{\dagger}-c_{3 j+2}^{\dagger}+c_{3 j+3}^{\dagger}, \\
\alpha_{2, \kappa}^{\dagger} & =\frac{1}{\sqrt{2 \mathcal{N}}} \sum_{j=0}^{\mathcal{N}-1} e^{-i \kappa j}\left(c_{3 j+1}^{\dagger}-c_{3 j+2}^{\dagger}\right), \\
\alpha_{3, \kappa}^{\dagger} & =\frac{1}{2 \sqrt{\mathcal{N}(2+\cos \kappa)}} \sum_{j=0}^{\mathcal{N}-1} e^{-i \kappa j} \\
& \times\left(c_{3 j-2}^{\dagger}+c_{3 j-1}^{\dagger}+2 c_{3 j}^{\dagger}+c_{3 j+1}^{\dagger}+c_{3 j+2}^{\dagger}\right) .
\end{aligned}
$$

Owing to the $\mathcal{N}$-fold degeneracy of the lowest flat band, one can use alternatively the states $l_{3 j}^{\dagger}|0\rangle, j=$ $0,1, \ldots, \mathcal{N}-1$ instead of the $\mathcal{N}$ states $\alpha_{1, \kappa}^{\dagger}|0\rangle$. These eigenstates are localized states where an electron is trapped on a diamond consisting of four sites, $3 j, 3 j+1$, $3 j+3$, and $3 j+2$ ( $j$ enumerates these diamond traps and varies from 0 to $\mathcal{N}-1)$. It is easy to check that $\left[H_{0}+\mu \sum_{i} n_{i}, l_{3 j}^{\dagger}\right]=\varepsilon_{1} l_{3 j}^{\dagger}$. A real-space picture for the state with $\kappa=\pi$ from the dispersive band $\varepsilon_{2}(\kappa)$ is as follows:

$$
\alpha_{2, \pi}^{\dagger}|0\rangle=\frac{1}{\sqrt{2 \mathcal{N}}} \sum_{j=0}^{\mathcal{N}-1}(-1)^{j}\left(c_{3 j+1}^{\dagger}-c_{3 j+2}^{\dagger}\right)|0\rangle .
$$

This eigenstate is not localized on a finite region, but the electron is trapped on the two legs [the sites inside the strip (i.e., numbers $3 j, 3 j+3$, etc.) do not appear in $\alpha_{2, \pi}^{\dagger}|0\rangle$. In what follows we call the state $\alpha_{2, \pi}^{\dagger}|0\rangle$ the trapped two-leg state. We may also introduce upper- and lower-leg states

$$
\begin{aligned}
L_{u}^{\dagger}|0\rangle & =\frac{1}{\sqrt{2}}\left(\alpha_{2, \pi}^{\dagger}-\lambda^{\dagger}\right)|0\rangle, \\
L_{l}^{\dagger}|0\rangle & =\frac{1}{\sqrt{2}}\left(\alpha_{2, \pi}^{\dagger}+\lambda^{\dagger}\right)|0\rangle, \\
\lambda^{\dagger} & =\frac{1}{\sqrt{2 \mathcal{N}}} \sum_{j=0}^{\mathcal{N}-1}(-1)^{j} l_{3 j}^{\dagger} .
\end{aligned}
$$

Obviously, the electron in the eigenstate $L_{u}^{\dagger}|0\rangle\left(L_{l}^{\dagger}|0\rangle\right)$ is trapped on the upper (lower) leg.

\section{Kagomé chain II}

Finally, we consider the kagomé chain II (Fig. 17) which consists of $\mathcal{N}=N / 5$ cells, each cell contains five sites. Again standard transformations lead to the diagonal form of the tight-binding Hamiltonian

$$
\begin{aligned}
H_{0}+\mu \sum_{i} n_{i} & =\sum_{p=1}^{5} \sum_{\kappa} \varepsilon_{p}(\kappa) \alpha_{p, \kappa}^{\dagger} \alpha_{p, \kappa}, \\
\varepsilon_{1}(\kappa) & =-2 t+\mu, \\
\varepsilon_{2}(\kappa) & =-t \sqrt{2+2 \cos \kappa}+\mu, \\
\varepsilon_{3}(\kappa) & =t-t \sqrt{3+2 \cos \kappa}+\mu, \\
\varepsilon_{4}(\kappa) & =t \sqrt{2+2 \cos \kappa}+\mu \\
\varepsilon_{5}(\kappa) & =t+t \sqrt{3+2 \cos \kappa}+\mu
\end{aligned}
$$

with $\kappa=2 \pi m / \mathcal{N}, m \in \mathbb{Z},-\mathcal{N} / 2<m \leq \mathcal{N} / 2$. The state with the energy $\varepsilon_{2}(\kappa=0)$ (it does exist for odd and even $\mathcal{N}$ ) touches the flat band. The $\kappa$-dependent singleelectron states are given by $\alpha_{p, \kappa}^{\dagger}|0\rangle, p=1,2,3,4,5$. For the sake of brevity we give $\alpha_{1, \kappa}^{\dagger}|0\rangle$ for the lowest-energy band, only

$$
\begin{array}{r}
\alpha_{1, \kappa}^{\dagger}=\frac{1}{\sqrt{2 \mathcal{N}(3-\cos \kappa)}} \sum_{j=0}^{\mathcal{N}-1} e^{-i \kappa j} l_{5 j}^{\dagger}, \\
l_{5 j}^{\dagger}=c_{5 j}^{\dagger}-c_{5 j+1}^{\dagger}-c_{5 j+2}^{\dagger}+c_{5 j+3}^{\dagger}+c_{5 j+4}^{\dagger}-c_{5 j+5}^{\dagger} .
\end{array}
$$

The corresponding localized states are given by $l_{5 j}^{\dagger}|0\rangle$, $j=0,1, \ldots, \mathcal{N}-1$, where an electron is trapped on a hexagon consisting of six sites, $5 j, 5 j+1,5 j+3,5 j+$ $5,5 j+4,5 j+2(j$ enumerates these hexagon traps and varies from 0 to $\mathcal{N}-1)$. It is easily verified that $\left[H_{0}+\right.$ $\left.\mu \sum_{i} n_{i}, l_{5 j}^{\dagger}\right]=\varepsilon_{1} l_{5 j}^{\dagger}$. A real-space picture for the state $\kappa=0$ from the dispersive band $\varepsilon_{2}(\kappa)$ is given by

$\alpha_{2,0}^{\dagger}|0\rangle=\frac{1}{2 \sqrt{\mathcal{N}}} \sum_{j=0}^{\mathcal{N}-1}\left(c_{5 j+1}^{\dagger}-c_{5 j+2}^{\dagger}-c_{5 j+3}^{\dagger}+c_{5 j+4}^{\dagger}\right)|0\rangle$.

As for the kagomé chain I the electron in this eigenstate is trapped on the two legs. Again in what follows we 
call the state $\alpha_{2,0}^{\dagger}|0\rangle$ the trapped two-leg state. Again we may introduce upper- and lower-leg states

$$
\begin{aligned}
L_{u}^{\dagger}|0\rangle & =\frac{1}{\sqrt{2}}\left(\alpha_{2,0}^{\dagger}-\lambda^{\dagger}\right)|0\rangle, \\
L_{l}^{\dagger}|0\rangle & =\frac{1}{\sqrt{2}}\left(\alpha_{2,0}^{\dagger}+\lambda^{\dagger}\right)|0\rangle, \\
\lambda^{\dagger} & =\frac{1}{2 \sqrt{\mathcal{N}}} \sum_{j=0}^{\mathcal{N}-1} l_{5 j}^{\dagger} .
\end{aligned}
$$

Obviously, the electron in the eigenstate $L_{u}^{\dagger}|0\rangle\left(L_{l}^{\dagger}|0\rangle\right)$ is trapped on the upper (lower) leg.

\section{Trapped states and destructive interference. The geometrical perspective}

It is useful to discuss the appearance of localized electron states from a geometrical point of view. As discussed above, for all three lattices we can easily single out a small area of the lattice which plays the role of a "trapping cell", namely a V-valley for the sawtooth chain, a diamond for the kagomé chain I, and a hexagon for the kagomé chain II (see marked regions in Fig. 1). Solving the single-electron problem for the trap one finds the lowest-energy eigenfunction $\propto \sum_{i} a_{i} c_{i}^{\dagger}|0\rangle$ with $a_{1}=1$, $a_{2}=-\sqrt{2}, a_{3}=1$ (sawtooth chain, the corresponding energy is $\left.-\sqrt{2} t^{\prime}<0\right), a_{1}=-a_{2}=a_{3}=-a_{4}=1$ (kagomé chain I, the corresponding energy is $-2 t<0$ ), or $a_{1}=-a_{2}=a_{3}=-a_{4}=a_{5}=-a_{6}=1$ (kagomé chain II, the corresponding energy is $-2 t<0)$. A crucial point is that the scheme of the bonds connecting the trapping cell with the rest should prevent the escape of the localized electron from the trap, i.e., the constructed oneelectron (localized) state should remain an eigenstate of the Hamiltonian (1.1) on the infinite lattice. It is easy to show that a sufficient condition for this is $\sum_{i} t_{r, i} a_{i}=0$, where the sum runs over all sites $i$ of a trapping cell and $r$ is an arbitrary site which does not belong to the trap, see also Refs. 26 and 12 . Indeed, the above condition is fulfilled if an arbitrary bond belonging to the trap and the bonds attached to the two sites of this bond in the trap form such a triangle that the electron amplitude on all sites outside the trapping cell is zero (destructive quantum interference). Note that a similar localization mechanism can be caused by a magnetic field for tightbinding electrons in two-dimensional structures, where the wave packet is bounded in Aharonov-Bohm cages due to destructive interference for particular values of the magnetic flux. 47

Interestingly, also the (extended) upper- and lower-leg states (3.6), (3.10) fit to this geometrical picture, if we interpret each of the two legs as a regular polygon (with an even number of sites). Then again two neighboring sites of the polygon are surrounded by equilateral triangles which prevent the electron to escape from the leg. Thus, for the kagomé chains we have $\mathcal{N}$ localized states located on diamonds or hexagons and, in addition, two states trapped on the legs (upper-leg state and lower-leg state), i.e., in total $\mathcal{N}+2$ localized states. (Note that for the sawtooth chain such additional states do not exist.) However, these $\mathcal{N}+2$ localized states are not linearly independent, since there is one linear relation between them, $L_{l}^{\dagger}|0\rangle-L_{u}^{\dagger}|0\rangle=\sqrt{2} \lambda^{\dagger}|0\rangle$, where $L_{l}^{\dagger}, L_{u}^{\dagger}$, and $\lambda^{\dagger}$ are given in Eq. (3.6) or in Eq. (3.10) (see also the general discussion of linear independence in Appendix B . As a result, there are only $\mathcal{N}+1$ linearly independent localized single-electron states. These simple arguments are in perfect agreement with the more detailed calculations presented in Sec. IIB and Sec. IIC.

\section{TRAPPED ELECTRON GROUND STATES FOR $U>0$}

In the previous section, we have found simple highly degenerate localized one-particle ground states of noninteracting spinless electrons which are created by operators $l_{2 j}^{\dagger}, l_{3 j}^{\dagger}$, or $l_{5 j}^{\dagger}$, see Eqs. (3.2), (3.4), or (3.8) [and also by $\alpha_{2, \pi}^{\dagger}$ or $\alpha_{2,0}^{\dagger}$ operators for the kagomé chains, see Eqs. (3.5) or (3.9)]. It is straightforward to create a set of $n$-electron ground states with $1<n \leq \mathcal{N}$ for the sawtooth chain and $1<n \leq \mathcal{N}+1$ for the kagomé chains by applying $n$ different (i.e., attached to different trapping cells) operators $l_{2 j}^{\dagger}, l_{3 j}^{\dagger}$, or $l_{5 j}^{\dagger}$ [for the kagomé chain I (II) we may also apply the operator $\left.\alpha_{2, \pi}^{\dagger}\left(\alpha_{2,0}^{\dagger}\right)\right]$. The energy of these states is $n \varepsilon_{1}$, and their degeneracy grows exponentially with the system size.

Now we return to the spinful case of interacting electrons, i.e., $U>0$ in Eq. (1.1). Clearly the inclusion of the spin does not change the energy for $U=0$ but increases the degeneracy. Let us denote the degeneracy at $U=0$ of the ground states of $n$ electrons by $g_{\mathcal{N}}^{(0)}(n)$. Obviously, one has $g_{\mathcal{N}}^{(0)}(n)=\left(\begin{array}{c}2 n_{\max } \\ n\end{array}\right), n \leq n_{\max }$, where $n_{\max }=\mathcal{N}$ for the sawtooth chain and odd- $\mathcal{N}$ kagomé chain I, but $n_{\max }=\mathcal{N}+1$ for even- $\mathcal{N}$ kagomé chain I and kagomé chain II.

First of all we note that the Hubbard interaction in Eq. (1.1) is a positive semidefinite operator and hence it can only increase the eigenvalues of the Hamiltonian (1.1). On the other hand, among the huge number of localized $n$-electron states being ground states for $U=0$ there is a considerable fraction of states which do not feel the Hubbard interaction term and thus they remain ground states with the $U$-independent energy $n \varepsilon_{1}$ for $U>0$. However, it is evident that the ground-state degeneracy, $g_{\mathcal{N}}(n)$, should decrease as $U>0$ is switched on, i.e., $g_{\mathcal{N}}(n)<g_{\mathcal{N}}^{(0)}(n)$. We will consider the cases of the sawtooth chain and of the kagomé chains in more detail separately. 


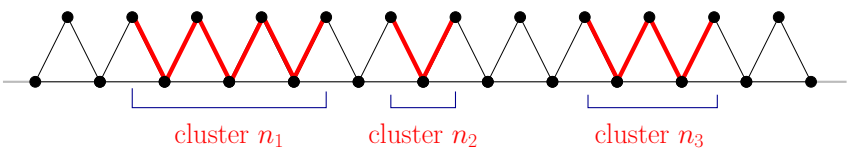

FIG. 2: (Color online) Illustration of a multi-cluster state. For each cluster the total spin of the cluster can be flipped independently by applying the cluster spin-flip operator $S_{n_{i}}^{-}=$ $\sum_{j \in \text { cluster } n_{i}} c_{j, \downarrow}^{\dagger} c_{j, \uparrow}$.

\section{A. Sawtooth chain and localized ground states}

Let us recall that for a localized state an electron with arbitrary spin $\sigma=\uparrow, \downarrow$ is trapped on three contiguous sites (V-shaped trapping cell). It is evident that $n$-electron states where the electrons (independently of their spins) are located in disconnected V-valleys (i.e., V-valleys without common sites) are ground states in the $n$-electron subspace having the energy $n \varepsilon_{1}$. The explicit expression for this type of ground states reads: $l_{2 j_{1}, \sigma_{1}}^{\dagger} \ldots l_{2 j_{n}, \sigma_{n}}^{\dagger}|0\rangle$, $\forall\left|j_{k}-j_{s}\right| \geq 2$.

However, these states do not exhaust all ground states in the subspace with $n$ electrons. Another type of ground states consists of $n$ trapped electrons all with identical spin $\sigma$ occupying a cluster of $n$ contiguous $\mathrm{V}$-valleys, e.g.,

$$
l_{2 j_{1}, \uparrow}^{\dagger} \ldots l_{2\left(j_{1}+n-1\right), \uparrow}^{\dagger}|0\rangle .
$$

Since the interaction term is inactive, this is still an eigenstate of the Hamiltonian (1.1) with eigenvalue $n \varepsilon_{1}$. Now we take into account the $\mathrm{SU}(2)$-invariance of the Hubbard model (1.1), i.e., $\left[S^{-}, H\right]=\left[S^{+}, H\right]=\left[S^{z}, H\right]=0$, where

$$
\begin{aligned}
S^{-} & =\sum_{i} c_{i, \downarrow}^{\dagger} c_{i, \uparrow}, \\
S^{+} & =\sum_{i} c_{i, \uparrow}^{\dagger} c_{i, \downarrow}, \\
S^{z} & =\frac{1}{2} \sum_{i}\left(c_{i, \uparrow}^{\dagger} c_{i, \uparrow}-c_{i, \downarrow}^{\dagger} c_{i, \downarrow}\right) .
\end{aligned}
$$

Repeated application of the spin-lowering operator $S^{-}$to the state (4.1) yields $2 s+1=n+1$ components of the $\operatorname{spin}-(n / 2)$ multiplet

$$
\left(S^{-}\right)^{m} l_{2 j_{1}, \uparrow}^{\dagger} \ldots l_{2\left(j_{1}+n-1\right), \uparrow}^{\dagger}|0\rangle, \quad m=0,1, \ldots, n .
$$

Evidently, all these states have the same energy $n \varepsilon_{1}$. Using the commutation relation

$$
\left[S^{-}, l_{2 j, \uparrow}^{\dagger}\right]=l_{2 j, \downarrow}^{\dagger}
$$

one can easily write down the explicit expressions for the states (4.3) in terms of operators $l_{2 j}^{\dagger}$ only.

To construct the remaining ground states in the subspace with $n$ electrons with the energy $n \varepsilon_{1}$ we consider now multi-cluster states $\stackrel{11}{ }$ We consider all possible splittings of $n$ into a sum $n=n_{1}+n_{2}+\ldots ; n_{i}>0$ such that clusters of $n_{i}$ consecutive occupied $\mathrm{V}$-valleys are separated by at least one empty valley, see Fig. 2 for an example. Starting from a fully spin-polarized multi-cluster state we can now independently act with the cluster spinlowering operators, $S_{n_{i}}^{-}=\sum_{j \in \text { cluster } n_{i}} c_{j, \downarrow}^{\dagger} c_{j, \uparrow}$, where $j$ runs over all sites in the cluster $n_{i}$. This yields manyelectron ground states which are products of the multiplet components in each cluster.

In order to compute the ground-state degeneracy $g_{\mathcal{N}}(n)$, we now need to compute the number $D_{\mathcal{N}}(n)$ of states which are constructed in the manner which we just described. This is complicated a bit by the non-trivial components of the $\mathrm{SU}(2)$-multiplets consisting of linear combinations of products of localized many-electron states. However, all coefficients of these linear combinations are positive and one can choose one state to represent the complete linear combination, for example, the one where all spins $\sigma=\uparrow$ are at the left of each $n$ contiguously occupied V-valleys and the $\sigma=\downarrow$ are at the right of the clusters 11 This reduces the counting problem to counting the number of configurations $\mathcal{Z}(n, \mathcal{N})$ of three states in the trapping cells, namely empty (0) and occupied with $\sigma=\uparrow, \downarrow$, subject to the constraint that no $\uparrow$-state is allowed to appear as the right neighbor of a $\downarrow$-state. This combinatorial problem can be solved directly with a $3 \times 3$ transfer matrix, yielding the canonical partition functions $\mathcal{Z}(n, \mathcal{N})$ (see Appendix $\mathrm{A}$ ). One small additional step then yields $D_{\mathcal{N}}(n)$ : in the sector with $n=\mathcal{N}$ and for periodic boundary conditions, there are only two allowed configurations according to the rules which we just described whereas the $\mathrm{SU}(2)$ multiplet has $\mathcal{N}+1$ components (Tasaki's ferromagnetic ground states). Hence, in the sector with $n=\mathcal{N}$ and for periodic boundary conditions we need to add $\mathcal{N}-1$ configurations by hand. Putting all this together, we obtain the ground-state degeneracy of the many-electron configurations with $n \leq \mathcal{N}$ electrons localized in $\mathcal{N}$ traps

$$
\begin{aligned}
g_{\mathcal{N}}(n) & =D_{\mathcal{N}}(n), \\
D_{\mathcal{N}}(n) & =\mathcal{Z}(n, \mathcal{N})+(\mathcal{N}-1) \delta_{n, \mathcal{N}} .
\end{aligned}
$$

As described above, the last term, $(\mathcal{N}-1) \delta_{n, \mathcal{N}}$, ensures the correct counting in the sector $n=\mathcal{N}$. Note furthermore that by writing $g_{\mathcal{N}}(n)=D_{\mathcal{N}}(n)$ we have implicitly assumed that the construction sketched at the beginning of this sections yields all ground states. This is indeed the case for the sawtooth chain, 11 see also below.

An alternative way to compute $\mathcal{Z}(n, \mathcal{N})$ has been described in Ref. 11: the combinatorial problem of the three states $0, \sigma=\uparrow, \downarrow$ in $\mathcal{N}$ traps can be mapped to a harddimer problem on an auxiliary simple chain with $2 \mathcal{N}$ sites. In this mapping, one associates 2 sites to each Vvalley in order to accommodate either one spin projection such that the aforementioned constraint that no $\uparrow$-state is allowed to appear as the right neighbor of a $\downarrow$-state and the constraint that double-occupancy of a $\mathrm{V}$-valley is forbidden map to hard-dimer exclusion rules. This auxiliary 
hard-dimer problem on $2 \mathcal{N}$ sites has been solved with a $2 \times 2$ transfer matrix ${ }^{11}$ and therefore we may also refer to the trapped electron configurations as "hard-dimer configurations". Of course, the different ways of computing $\mathcal{Z}(n, \mathcal{N})$ are completely equivalent.

In what follows, we call many-electron ground states, which are constructed only from the localized singleelectron states trapped in a V-valley "hard-dimer states". Their degeneracy $D_{\mathcal{N}}(n)$ is given by the second line of Eq. (4.5). The hard-dimer states are the only ground states for the sawtooth chain and for the kagomé chain I with an odd number of cells $\mathcal{N}$. For the kagomé chain I with an even number of cells $\mathcal{N}$ as well as for the kagomé chain II in addition to the hard-dimer states we have to consider also many-electron ground states which involve an extended single-electron state with the flat-band energy $\varepsilon_{1}$ - the two-leg state [see Eqs. (3.5) and (3.9)].

\section{B. Kagomé chains and trapped ground states involving two-leg states}

Now we consider the ground states in the subspace with $n=1,2 \ldots, \mathcal{N}+1$ electrons for the case when the two-leg state comes into play (periodic kagomé chain I with even number of cells $\mathcal{N}$ or periodic kagomé chain II). Repeating the arguments elaborated for the sawtooth chain, for $n=1,2, \ldots, \mathcal{N}$ we can construct hard-dimer states the number of which is given in Eq. (4.5). However, the hard-dimer states do not exhaust all many-electron ground states for $n=1,2, \ldots, \mathcal{N}$. Indeed, in the subspace with $n=1$ electron we have in addition two ground states $\alpha_{2, \pi, \sigma}^{\dagger}|0\rangle, \sigma=\uparrow, \downarrow$ for the kagomé chain I [see Eq. (3.5)] or $\alpha_{2,0, \sigma}^{\dagger}|0\rangle, \sigma=\uparrow, \downarrow$ for the kagomé chain II [see Eq. (3.9)].

What happens if $n>1$ ? Consider the case $n=2$. We can construct ground states using the two-leg state as follows. Assume both electrons have the same spin polarization and the first one is localized within 1 of $\mathcal{N}$ (diamond or hexagon) cells whereas the second one is in a two-leg state. Obviously this is the ground state with the energy $2 \varepsilon_{1}$. More states can be generated applying $S^{ \pm}$two times. Thus, the degeneracy of the constructed ground state is $3 \mathcal{N}$.

Interestingly, in the subspace with $n=2$ electrons there is one more possibility to construct an eigenstate of the interacting Hamiltonian (1.1) with the energy $2 \varepsilon_{1}$. Recall that the electron in the state $L_{u \sigma}^{\dagger}|0\rangle$ [see Eq. (3.6) or Eq. [3.10) is located along the upper leg, whereas the electron in the state $L_{l \sigma}^{\dagger}|0\rangle$ [see Eq. (3.6) or Eq. (3.10)] is located along the lower leg. Therefore, even if two electrons being in these states have opposite spins, they do not feel the Hubbard repulsion (no common sites) and the energy of this two-electron state remains $2 \varepsilon_{1}$. Thus, we find the following (extra) state in the subspace with $n=2$ electrons for the periodic kagomé chains:

$$
\mid \text { extra }\rangle=L_{l \sigma}^{\dagger} L_{u,-\sigma}^{\dagger}|0\rangle
$$

where $L_{u \sigma}^{\dagger}, L_{l \sigma}^{\dagger}$ are defined by Eq. (3.6) for the periodic kagomé chain I and by Eq. (3.10) for the periodic kagomé chain II.

Consider next the subspace with $n=3$ electrons. Again we can assume three electrons to have the same spin, two of which are localized within 2 of $\mathcal{N}$ cells whereas the third one is in the two-leg state with the flat-band energy. Obviously this is the ground state with the energy $3 \varepsilon_{1}$. More states can be generated applying $S^{ \pm}$three times. Thus, the degeneracy of the constructed ground state is $4\left(\begin{array}{c}\mathcal{N} \\ 2\end{array}\right)$. Proceeding with such arguments for $n=4, \ldots, \mathcal{N}+1$ electrons we can easily construct the ground states which involve the two-leg state. Obviously, it is easy to count their number which is equal to $(n+1)\left(\begin{array}{c}\mathcal{N} \\ n-1\end{array}\right)$.

We wish to emphasize here that for the kagomé chains (kagomé I with even $\mathcal{N}$ or kagomé II chains) we have constructed the ground state in the subspace with $n=$ $\mathcal{N}+1$ electrons, which has the energy $(\mathcal{N}+1) \varepsilon_{-}$, starting from the fully polarized state with $\mathcal{N}$ electrons occupying diamond or hexagon trapping cells, respectively, and 1 electron being in the two-leg state, and then applying the $S^{ \pm}$operator $\mathcal{N}+1$ times. The degeneracy of the constructed ground state is $\mathcal{N}+2$.

Summing up, we have arrived at the following formula for the degeneracy of the ground states in the subspaces with $n \leq \mathcal{N}+1$ electrons for the periodic kagomé chain I with an even number of cells $\mathcal{N}$ or the periodic kagomé chain II:

$$
\begin{aligned}
& g_{\mathcal{N}}(n)=\left(1-\delta_{n, \mathcal{N}+1}\right) D_{\mathcal{N}}(n)+\left(1-\delta_{n, 0}\right) L_{\mathcal{N}}(n) \\
& L_{\mathcal{N}}(n)=(n+1)\left(\begin{array}{c}
\mathcal{N} \\
n-1
\end{array}\right)+\delta_{n, 2}
\end{aligned}
$$

$\left[n=0,1, \ldots, \mathcal{N}\right.$ for $D_{\mathcal{N}}(n)$, whereas $n=1, \ldots, \mathcal{N}+1$ for $\left.L_{\mathcal{N}}(n)\right]$.

\section{Some properties of the ground states for $n \leq n_{\max }$}

As we have explained above, the ground states in the subspaces with $n \leq n_{\max }$ electrons can be constructed either from hard-dimer states only (sawtooth, kagomé I with odd $\mathcal{N}, n_{\max }=\mathcal{N}$ ) or from hard-dimer states and the two-leg state (kagomé I with even $\mathcal{N}$, kagomé II, $n_{\max }=\mathcal{N}+1$ ) and the degeneracy of the ground state $g_{\mathcal{N}}(n)$ in the subspace with $n \leq n_{\max }$ electrons can be determined either according to Eq. (4.5) (sawtooth, kagomé I with odd $\mathcal{N}$ ) or according to Eq. (4.7) (kagomé I with even $\mathcal{N}$, kagomé II). For the calculation of $\mathcal{Z}(n, \mathcal{N})$, see Appendix A

Fig. 3 illustrates the difference in the ground-state degeneracy $g_{\mathcal{N}}(n)$ conditioned by the ground states involving two-leg states. We take as examples $\mathcal{N}=7$ and 8. The sawtooth chain never has two-leg state contributions, the kagomé chain II always has two-leg state contributions, and for the kagomé chain I the two-leg state 


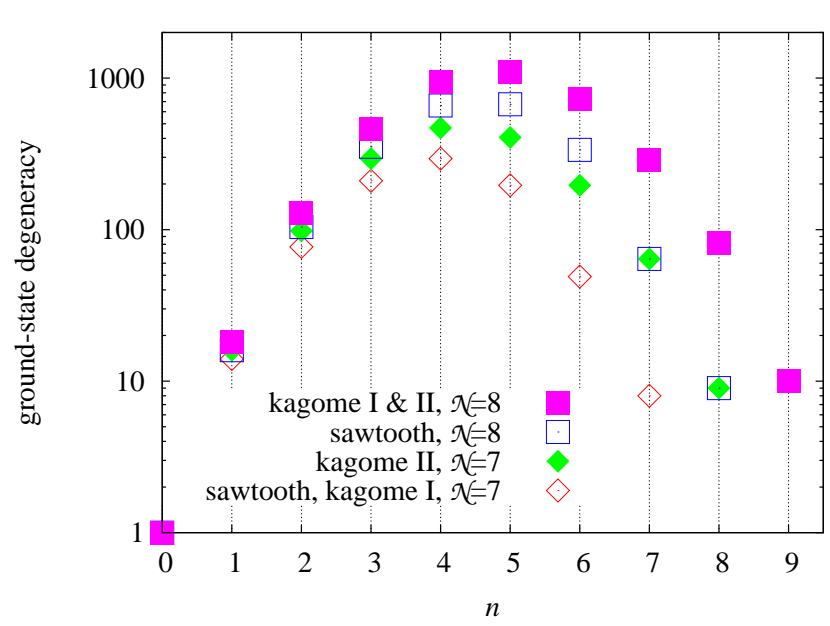

FIG. 3: (Color online) Ground-state degeneracies for $n=$ $1, \ldots, n_{\max }$ for the sawtooth chain $(\mathcal{N}=7$, empty diamonds; $\mathcal{N}=8$, empty squares), the kagomé chain I $(\mathcal{N}=7$, empty diamonds; $\mathcal{N}=8$, filled squares), and the kagomé chain II $(\mathcal{N}=7$, filled diamonds; $\mathcal{N}=8$, filled squares $)$.

contributions appear only for even $\mathcal{N}$. Consequently, for $\mathcal{N}=8$, the degeneracies of the two kagomé chains are identical whereas for $\mathcal{N}=7$ the degeneracy of the kagomé chain I is identical to that of the sawtooth chain. Firstly, we observe that the degeneracies increase rapidly with $\mathcal{N}$ (note the logarithmic scale of the vertical axis of Fig. 3). Secondly, there is an obvious contribution of the two-leg states.

Next, we discuss some general properties of the constructed many-electron ground states in the subspaces with $n \leq n_{\max }$ electrons. As we have mentioned already, these states having the energy $n \varepsilon_{1}$ as in the case $U=0$ are indeed the lowest-energy (ground) states for $U>0$ since the Hubbard interaction term $H_{\text {int }}=U \sum_{i} n_{i, \uparrow} n_{i, \downarrow}$ in Eq. (1.1) is a positive semidefinite operator and can only increase energies.

Another less simple question concerns completeness of the constructed ground states. In other words, are the constructed ground states the only ground states? Here we use numerics for finite systems to check completeness. Exact diagonalization data for the sawtooth chain with $N=6,8,10,12,14,16$, the kagomé chain I with $N=$ $9,12,15,18,21,24$, and the kagomé chain II with $N=$ $15,20,25,30$ are in perfect agreement with predictions of Sections IVA and IVB compare also Fig. 3. These numerical findings are supported by general arguments: We know that the localized states (including the two-leg states) are complete for $U=0$. Adding a finite $U$, a part of these states will have higher energy, but no new ground states will appear, since $U$ can only increase the energy.

We have also estimated the energy gap between the ground state and the first excited state $\Delta_{n}$ in the subspace with $n$ electrons. For $N \rightarrow \infty$ we have $\Delta_{1}=$ $\varepsilon_{2}(\pi)-\varepsilon_{1}=2 t$ (sawtooth chain) and $\Delta_{1}=0$ (kagomé chains). Assuming $U \rightarrow \infty$ we found for finite systems of $N=30$ sites $\Delta_{2} \approx 0.4044$ (sawtooth) but $\Delta_{2} \approx 0.0689$ (kagomé I) and $\Delta_{2} \approx 0.0584$ (kagomé II). These data give a hint that the excitation gap for finite kagomé chains is much smaller than for the sawtooth chain. A finite-size extrapolation to $N \rightarrow \infty$ for fixed electron density $n / N$ suggests a vanishing gap in the thermodynamic limit even for the sawtooth chain. Note, however, that in real materials with chain structure impurities and other lattice imperfections are always present and therefore one deals in practice with (an ensemble of) finite chains having a gap to the excited states.

With respect to the contribution of the trapped ground states (including the two-leg states) to thermodynamic quantities the question arises whether these states for a given $n \leq n_{\max }$ are linearly independent. Their linear independence can be shown following the lines of Ref. 48, for more details see Appendix B

\section{LOW-TEMPERATURE THERMODYNAMICS}

\section{A. Contribution of trapped electron states}

As shown in the preceding section, for all three onedimensional lattices considered the ground states of the Hubbard model (1.1) in the subspaces with $n=$ $0,1, \ldots, n_{\max }$ electrons have the energy $n \varepsilon_{1}$ and the degeneracy $g_{\mathcal{N}}(n)$ [see Eqs. (4.5), (4.7)]. Now if the chemical potential of electrons $\mu$ is around $\mu_{0}\left(\mu_{0}=2 t\right.$ for all three models) the constructed ground states in the subspaces with $n=0,1, \ldots, n_{\max }$ electrons will dominate the grand-canonical partition function at low temperatures due to their huge degeneracy, i.e.,

$$
\begin{aligned}
\Xi(T, \mu, N) & \approx \Xi_{\mathrm{GS}}(T, \mu, N) \\
& =\sum_{n=0}^{n_{\max }} g_{\mathcal{N}}(n) \exp \left(-\frac{n \varepsilon_{1}}{T}\right) \\
& =\sum_{n=0}^{n_{\max }} g_{\mathcal{N}}(n) \exp \left[\frac{n\left(\mu_{0}-\mu\right)}{T}\right] \\
& =\sum_{n=0}^{n_{\max }} g_{\mathcal{N}}(n) z^{n},
\end{aligned}
$$

where $z=\exp x$ is the activity and $x=\left(\mu_{0}-\mu\right) / T$.

Consider first the sawtooth chain and the kagomé chain I with an odd number of trapping cells $\mathcal{N}$, i.e., all ground states correspond to hard-dimer states. Inserting Eq. (4.5) into Eq. (5.1) we arrive at

$$
\begin{aligned}
\Xi_{\mathrm{GS}}(T, \mu, N) & =\Xi_{\text {trap }}(z, \mathcal{N})+(\mathcal{N}-1) z^{\mathcal{N}} \\
\Xi_{\text {trap }}(z, \mathcal{N}) & =\sum_{n=0}^{\mathcal{N}} z^{n} \mathcal{Z}(n, \mathcal{N}) .
\end{aligned}
$$


Calculating $\Xi_{\text {trap }}(z, \mathcal{N})$ with the help of the transfermatrix method ${ }^{49}$ (see Appendix $\mathrm{A}$ ) we get the following equations

$$
\begin{aligned}
\Xi_{\mathrm{GS}}(T, \mu, N) & =\xi_{+}^{\mathcal{N}}+\xi_{-}^{\mathcal{N}}+\xi_{3}^{\mathcal{N}}, \\
\xi_{ \pm} & =\left(\frac{1}{2} \pm \sqrt{\frac{1}{4}+z}\right)^{2}, \\
\xi_{3} & =(\mathcal{N}-1)^{\frac{1}{\mathcal{N}}} z .
\end{aligned}
$$

This is an important result, since it allows to calculate the contribution of all the ground states described by hard dimers to thermodynamic quantities explicitly.

Consider next the kagomé chain I with an even number of trapping cells $\mathcal{N}$ and the kagomé chain II. Insertion of Eq. (4.7) into Eq. (5.1) yields

$$
\begin{array}{r}
\Xi_{\mathrm{GS}}(T, \mu, N)=\Xi_{\text {trap }}(z, \mathcal{N})+(\mathcal{N}-1) z^{\mathcal{N}} \\
+\sum_{n=1}^{\mathcal{N}+1}(n+1)\left(\begin{array}{c}
\mathcal{N} \\
n-1
\end{array}\right) z^{n}+z^{2} .
\end{array}
$$

Here the first two terms account for the many-electron configurations associated to the $\mathcal{N}$ trapping cells, cf. Eq. (5.2), whereas the third and the fourth term are due to the two-leg state. After simple calculations we get the following final result

$$
\begin{array}{r}
\Xi_{\mathrm{GS}}(T, \mu, N)=\xi_{+}^{\mathcal{N}}+\xi_{-}^{\mathcal{N}}+\xi_{3}^{\mathcal{N}}+\xi_{4}^{\mathcal{N}}+\xi_{5}^{\mathcal{N}}+\xi_{6}^{\mathcal{N}}, \\
\xi_{4}=(2 z)^{\frac{1}{\mathcal{N}}}(1+z), \xi_{5}=\left(\mathcal{N} z^{2}\right)^{\frac{1}{\mathcal{N}}}(1+z)^{\frac{\mathcal{N}-1}{\mathcal{N}}}, \xi_{6}=z^{\frac{2}{\mathcal{N}}}
\end{array}
$$

$\left[\xi_{ \pm}\right.$and $\xi_{3}$ are defined in Eq. (5.3)].

The entropy $S(T, \mu, N)=-\partial \Omega(T, \mu, N) / \partial T$, the grand-canonical specific heat $C(T, \mu, N) \equiv$ $T \partial S(T, \mu, N) / \partial T$, and the average number of electrons $\bar{n}(T, \mu, N)=\partial \Omega(T, \mu, N) / \partial \mu$ follow from Eqs. (5.3), (5.5) and the formula for the grand-thermodynamical potential $\Omega(T, \mu, N)=-T \ln \Xi(T, \mu, N)$.

Although explicit formulas for these thermodynamic quantities for finite systems are too cumbersome to be written down explicitly here, it might be useful to consider some limiting cases:

(i) For $T=0$ and $\mu<\mu_{0}$ we have $z \rightarrow \infty$ and consequently we find for the residual entropy $S(\mu, N)=$ $\ln (\mathcal{N}+1)$ and $\bar{n}(\mu, N)=\mathcal{N}$ [hard-dimer states only, cf. Eq. [5.3)] or $S(\mu, N)=\ln (\mathcal{N}+2)$ and $\bar{n}(\mu, N)=\mathcal{N}+1$ [hard-dimer states and two-leg states, cf. Eq.(5.5)].

(ii) For $T=0$ and $\mu>\mu_{0}$ we have $z \rightarrow 0$ which leads to $S(\mu, N)=0$ and $\bar{n}(\mu, N)=0$.

(iii) At $\mu=\mu_{0}$ we have $z=1$ for all $T$ and we find for the residual entropy $S\left(\mu_{0}, N\right)=\ln \left\{[(3+\sqrt{5}) / 2]^{\mathcal{N}}+[(3-\right.$ $\left.\sqrt{5}) / 2]^{\mathcal{N}}+\mathcal{N}-1\right\}$ [hard-dimer states only, cf. Eq. [5.3)] or $S\left(\mu_{0}, N\right)=\ln \left\{[(3+\sqrt{5}) / 2]^{\mathcal{N}}+[(3-\sqrt{5}) / 2]^{\mathcal{N}}+\mathcal{N}+\right.$ $\left.2^{\mathcal{N}+1}+\mathcal{N} 2^{\mathcal{N}-1}\right\}$ [hard-dimer states and two-leg states, cf. Eq.(5.5)]. Moreover, $C\left(T, \mu_{0}, N\right)=0$ for any temperature independently of the system size.

Note, finally, that the conventional (canonical) specific

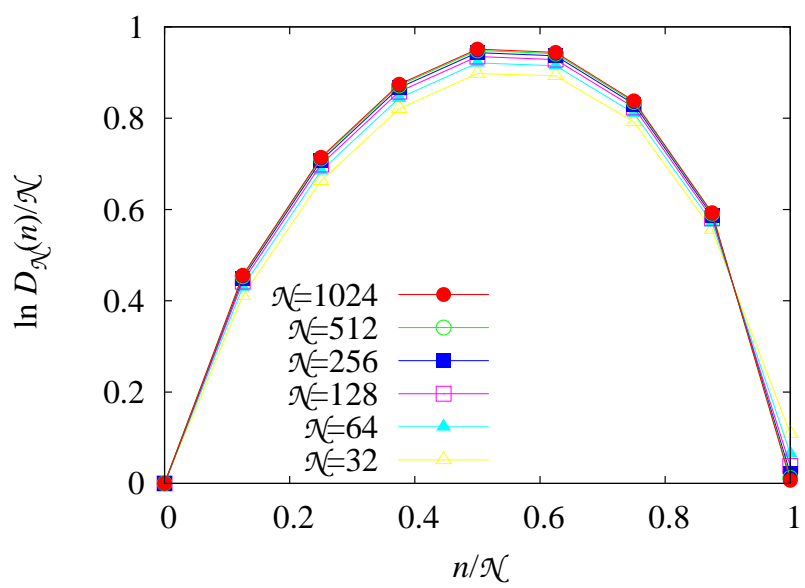

FIG. 4: (Color online) Canonical residual entropy $S(n, \mathcal{N}) / \mathcal{N}=\ln \left[D_{\mathcal{N}}(n)\right] / \mathcal{N}$ versus $n / \mathcal{N}$ for system sizes $\mathcal{N}=32,64,128,256,512,1024$.

heat $C(T, n, N)$ for a fixed number of electrons $n \leq n_{\max }$ is identically zero within the localized-state description.

In the thermodynamic limit $\mathcal{N} \rightarrow \infty$ only the largest term, i.e., $\xi_{+}^{\mathcal{N}}$, survives in the expressions (5.3) and (5.5) for the partition function ${ }^{50} \Xi_{\mathrm{GS}}(T, \mu, N)$ and we have

$$
\Xi_{\mathrm{GS}}(T, \mu, N)=\left(\frac{1}{2}+\sqrt{\frac{1}{4}+\exp \frac{2 t-\mu}{T}}\right)^{2 \mathcal{N}},
$$

which holds for all three considered one-dimensional lattices. The only differences consist in the relation between the number of cells $\mathcal{N}$ and the lattice size $N$ $(\mathcal{N}=N / 2, \mathcal{N}=N / 3$, and $\mathcal{N}=N / 5$ for the sawtooth chain, the kagomé chain I, and the kagomé chain II, respectively). Explicit formulas for these thermodynamic quantities can be obtained easily from the simple expression (5.6) for $\Xi_{\mathrm{GS}}(T, \mu, N)$. For instance, the residual entropy for $\mathcal{N} \rightarrow \infty$ is given by $S\left(\mu_{0}, N\right) / \mathcal{N}=$ $\ln [(3+\sqrt{5}) / 2]=0.96242 \ldots$, i.e., the ground-state degeneracy grows asymptotically with the system size according to $\varphi^{2 \mathcal{N}}$, where interestingly the golden mean $\varphi=(1+\sqrt{5}) / 2$ enters the expression.

Using the transfer-matrix representation we can also calculate numerically the (canonical) residual entropy $S(n, \mathcal{N}) / \mathcal{N}=\ln \left[D_{\mathcal{N}}(n)\right] / \mathcal{N}$ related to the hard-dimer states for certain fixed values of the electron number $n$ for $\mathcal{N}$ up to 1024. The results are shown in Fig. 4 . From Fig. 4 it is obvious that the largest degeneracy is found for $n$ around $\mathcal{N} / 2$. The extrapolation to $\mathcal{N} \rightarrow \infty$ yields $\lim _{\mathcal{N} \rightarrow \infty} \ln \left[D_{\mathcal{N}}(n=\mathcal{N} / 2)\right] / \mathcal{N} \approx 0.955$, which is very close to the grand-canonical residual entropy at $\mu=\mu_{0}$, see above. Indeed, the maximum of $\lim _{\mathcal{N} \rightarrow \infty} S(n, \mathcal{N}) / \mathcal{N}$ should reproduce the grand-canonical residual entropy, albeit with slow finite-size convergence. 


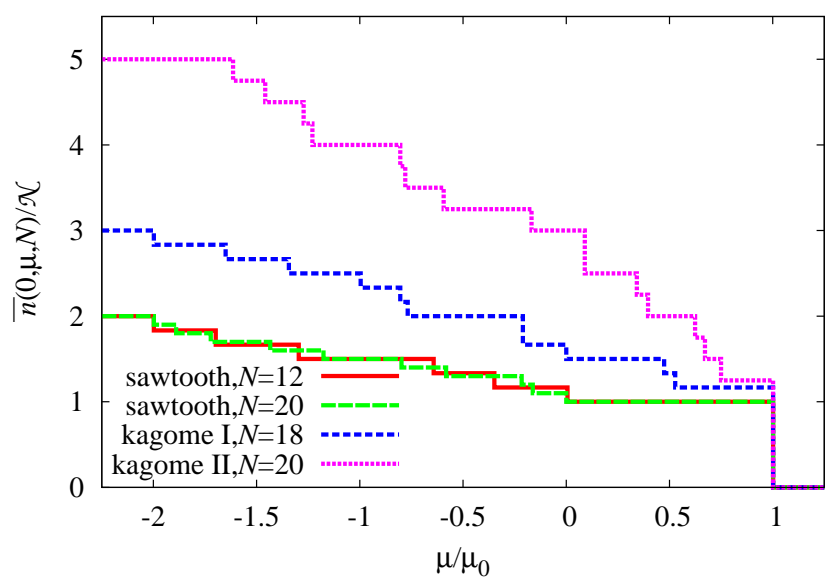

FIG. 5: (Color online) Average number of electrons in the ground state $\bar{n}(0, \mu, N) / \mathcal{N}$ versus chemical potential $\mu / \mu_{0}$ for the sawtooth chain $[N=12$ (solid), $N=20$ (long-dashed)], the kagomé chain I $[N=18$ (short-dashed)], the kagomé chain II $[N=20$ (dotted) $]$ with $U \rightarrow \infty$. The result for $\bar{n}(0, \mu, N) / \mathcal{N}$ which follows from Eqs. (5.3) and (5.5) is given by $\theta\left(1-\mu / \mu_{0}\right)$ and $[(\mathcal{N}+1) / \mathcal{N}] \theta\left(1-\mu / \mu_{0}\right)$, respectively.

\section{B. Comparison with exact diagonalization}

The above predictions are expected to be valid at low temperatures and for $\mu$ around $\mu_{0}$. In order to verify these expectations and examine the region of validity more precisely, we have performed complementary exact diagonalization of the full Hubbard Hamiltonian (1.1). First, we perform a symmetry reduction of the problem. In particular, we use separate conservation of the number of electrons with a given spin projection $n_{\uparrow}$ and $n_{\downarrow}$ as well as translational invariance. Ground states can then be obtained with the help of the Lanczos recursion. In order to obtain thermodynamic properties, we perform a full diagonalization in each symmetry subspace using a library routine. The problem simplifies a bit in the limit $U \rightarrow \infty$ where states with doubly occupied sites can be eliminated from the Hilbert space. Nevertheless, a full diagonalization of the complete problem can only be performed for small $N$. Low-temperature approximations for somewhat bigger $N$ can be obtained by omitting certain sectors of $n$.

Fig. 5 shows exact diagonalization data for the average number of electrons per cell $\bar{n}(T, \mu, N) / \mathcal{N}$ at $T=0$ as a function of the chemical potential $\mu / \mu_{0}$ for the sawtooth chain $(N=12,20)$, the kagomé chain I $(N=18)$, and the kagomé chain II $(N=20)$.

In Figs. 6] and 7 we show exact diagonalization data for the temperature dependence of the entropy per cell $S(T, \mu, N) / \mathcal{N}$ at $\mu=0.98 \mu_{0}, \mu_{0}, 1.02 \mu_{0}$ [panels (a)] and the temperature dependence of the grandcanonical specific heat per cell $C(T, \mu, N) / \mathcal{N}$ at $\mu=$ $0.98 \mu_{0}, \mu_{0}, 1.02 \mu_{0}$ [panels (b)] for the sawtooth chain
$(N=12)$ and the kagomé chain I $(N=9)$ (Fig. 6) and for the kagomé chain I $(N=12)$ and the kagomé chain II $(N=15)$ (Fig. 7) with $U \rightarrow \infty$. We also report analytical predictions for finite $\mathcal{N}$ [obtained from Eqs. (5.3) and (5.5)] as well for infinite $\mathcal{N}$ [obtained from Eq. (5.6)].

From Fig. 5 we see that in the ground state the average number of electrons per cell $\bar{n}(T, \mu, N) / \mathcal{N}$ as a function of $\mu / \mu_{0}$ exhibits a jump at $\mu / \mu_{0}=1$. This jump is related to the fact that all ground states with $0 \leq n \leq n_{\max }$ are degenerate at $\mu=\mu_{0}$. For $N \rightarrow \infty$ the jump is of height unity. For $\mu / \mu_{0}<1$ a plateau appears, see Fig. 5. The plateau width (i.e., the charge gap) increases as $U$ increases and rapidly approaches a saturation value for all three finite lattices (for the finite sawtooth chains with $N=12,16,20$ such data were reported in Fig. 1b of Ref. 11). What happens with the plateau width as $N$ increases? For the sawtooth chain the plateau width is almost independent of $N$ (compare the results for $N=$ 12, 16, 20 in Fig. 1b of Ref. 11). For $U \rightarrow \infty$ we can also find the exact ground state for $n=n_{\max }+1$, see Sec.VIC, which allows to determine the size-independent plateau width $\Delta \mu=2 t$. By contrast, for the kagomé chains the plateau disappears as $N \rightarrow \infty$.

From Figs. 6 and 7 (temperature dependences of entropy and specific heat) we see that analytical predictions for finite $\mathcal{N}$ as they follow from Eqs. (5.3) and (5.5) perfectly reproduce the exact diagonalization data at low temperatures. Note, however, that for finite $N$ the deviation from the hard-dimer description is noticeable, although in the thermodynamic limit $N \rightarrow \infty$ Eqs. (5.3) and (5.5) imply the one-dimensional hard-dimer behavior. Finite-size effects are clearly seen in the panels corresponding to $\mu=0.98 \mu_{0}$ (Figs. 6] and 7) and to $\mu=\mu_{0}$ and $\mu=1.02 \mu_{0}$ (Fig. 7). The most prominent features seen in these plots are a finite value of the entropy at very low temperatures for $\mu=\mu_{0}$ and an extra low-temperature maximum in the specific heat $C(T, \mu, N)$ for $\mu \neq \mu_{0}$. The value of the residual entropy for finite systems as it follows from analytical predictions based on Eqs. (5.3), (5.5) and exact diagonalization are in perfect agreement. A high-temperature maximum of $C(T, \mu, N) / \mathcal{N}$ around $T \approx \mu_{0}$ is common for any system with a finite bandwidth, whereas a low-temperature peak of $C(T, \mu, N) / \mathcal{N}$ around $T \approx 0.01 \mu_{0}$ for $\left|\mu-\mu_{0}\right|=0.02 \mu_{0}$ emerges due to the manifold of localized electron states. Indeed, the analytical predictions which follow from Eqs. (5.3), (5.5) for finite $\mathcal{N}$ are indistinguishable from the $U \rightarrow \infty$ exact diagonalization data in the low-temperature peak region. We should, however, mention that for $N \rightarrow \infty$ the excitation spectrum of the full Hubbard model in the many-electron sectors is most likely gapless, in particular for finite values of $U$ (compare Sec. IVC and Ref. 37b). Such gapless excitations could give rise to quantitative corrections at all temperatures for $\mu<\mu_{0}$. Indeed such quantitative deviations are visible for instance on finitesize sawtooth chains with $\mu=0.98 \mu_{0}$ and $U=4 t .11,37$

To demonstrate the effect of finite $U$ on temperature dependences we consider as an example the kagomé chain 

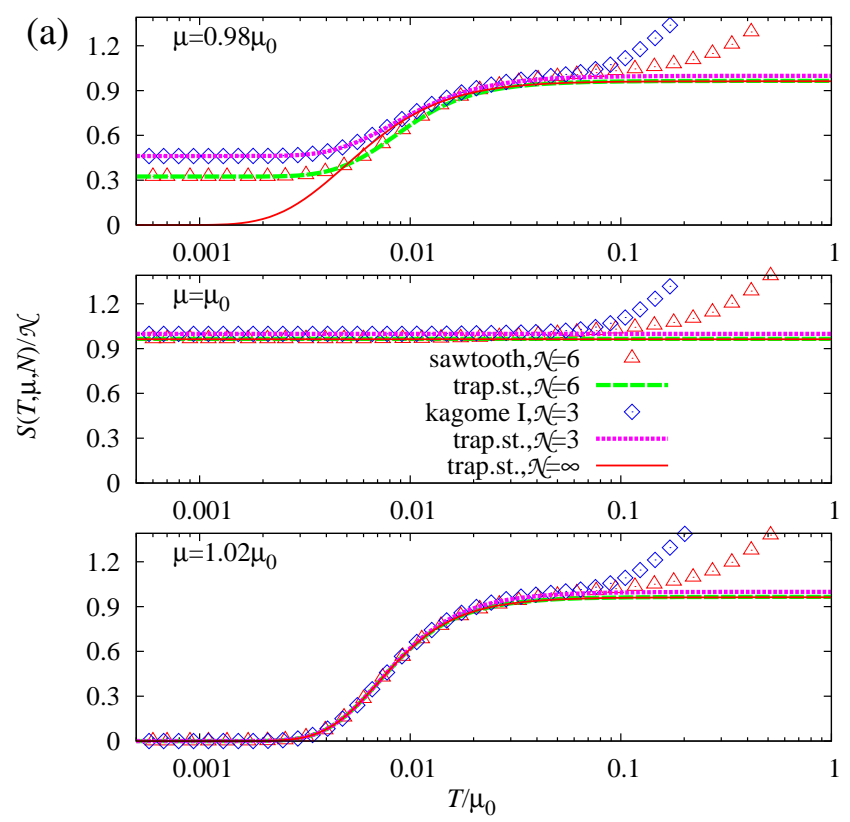

(b)
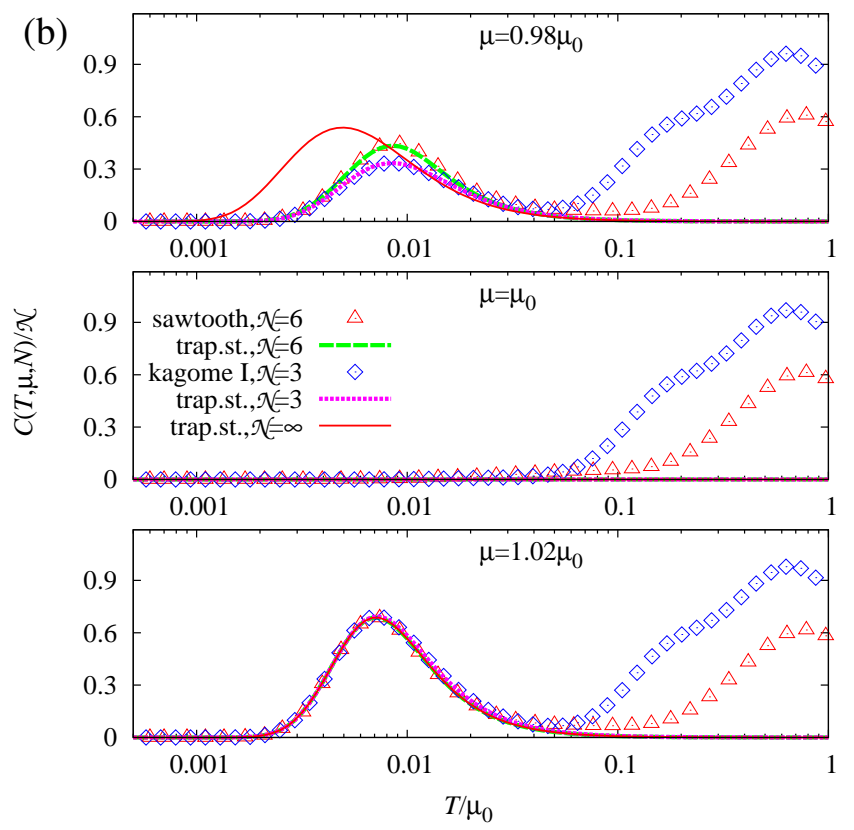

FIG. 6: (Color online) Entropy $S(T, \mu, N) / \mathcal{N}$ and grandcanonical specific heat $C(T, \mu, N) / \mathcal{N}$ for the sawtooth chain $(N=12$, triangles $)$ and the kagomé chains I $(N=9$, diamonds) with $t=1, U \rightarrow \infty$. (a) $S(T, \mu, N) / \mathcal{N}$ versus temperature $T / \mu_{0}$ at $\mu=0.98 \mu_{0}, \quad \mu_{0}, 1.02 \mu_{0}$ (from top to bottom). (b) $C(T, \mu, N) / \mathcal{N}$ versus temperature $T / \mu_{0}$ at $\mu=0.98 \mu_{0}, \mu_{0}, 1.02 \mu_{0}$ (from top to bottom). We also show the hard-dimer result for $N \rightarrow \infty$ as it follows from (5.6) (thin solid lines) as well as the results which follow from Eq. (5.3) for $\mathcal{N}=3$ (dotted lines) and $\mathcal{N}=6$ (dashed lines). Note that for these systems no additional leg states exist. Note further that for $\mu=\mu_{0}$ and $\mu=1.02 \mu_{0}$ the hard-dimer results for $\mathcal{N}=3,6$, and $\infty$ are indistinguishable.
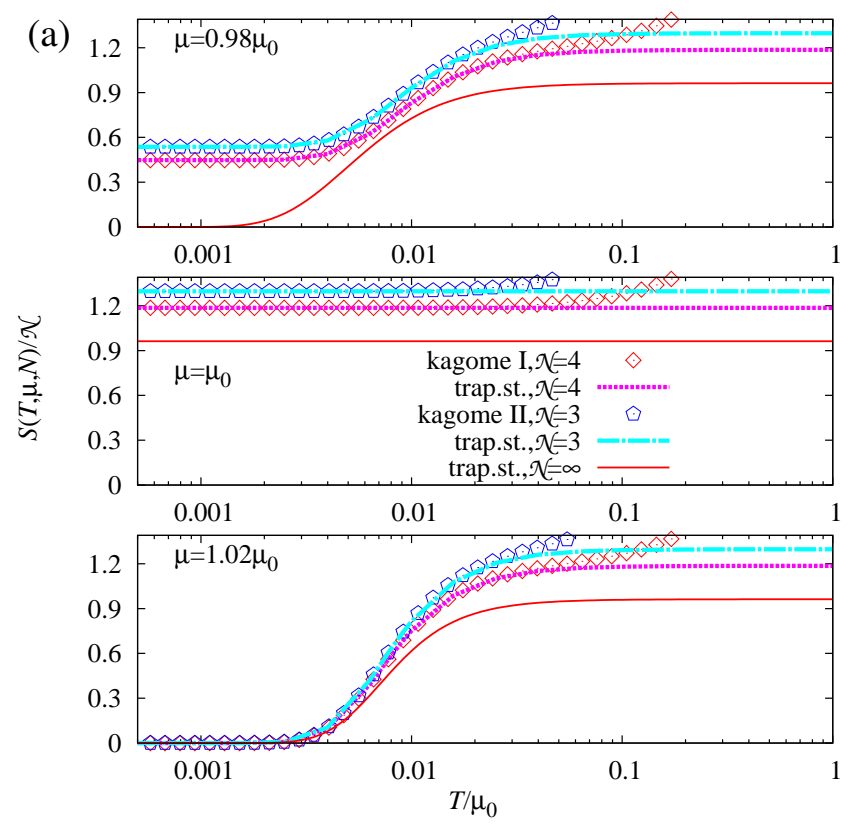

(b)
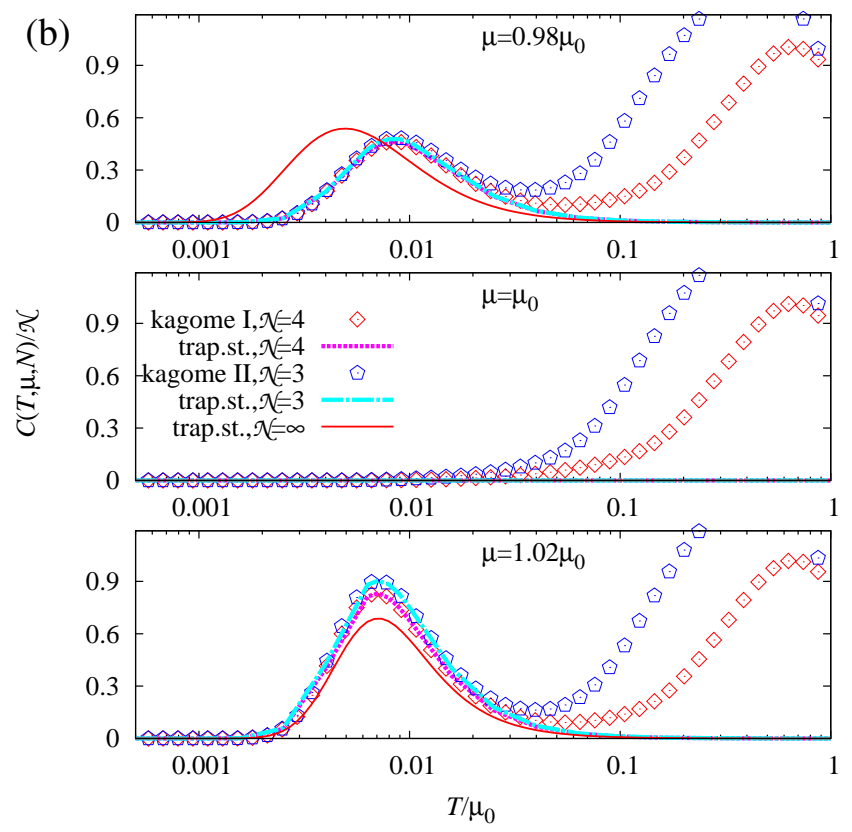

FIG. 7: (Color online) Entropy $S(T, \mu, N) / \mathcal{N}$ and grandcanonical specific heat $C(T, \mu, N) / \mathcal{N}$ for the kagomé chain I $(N=12$, diamonds $)$ and the kagomé chain II $(N=15$, the sectors with up to 7 electrons were taken into account, pentagons) with $t=1, U \rightarrow \infty$. (a) $S(T, \mu, N) / \mathcal{N}$ versus temperature $T / \mu_{0}$ at $\mu=0.98 \mu_{0}, \mu_{0}, 1.02 \mu_{0}$ (from top to bottom). (b) $C(T, \mu, N) / \mathcal{N}$ versus temperature $T / \mu_{0}$ at $\mu=0.98 \mu_{0}, \mu_{0}, 1.02 \mu_{0}$ (from top to bottom). We also show the hard-dimer result for $N \rightarrow \infty$ as it follows from (5.6) (thin solid lines) as well as the finite-size results for $\mathcal{N}=3$ (dashdotted lines) and $\mathcal{N}=4$ (dotted lines) which follow from Eq. (5.5) and include the contribution of the leg states. 

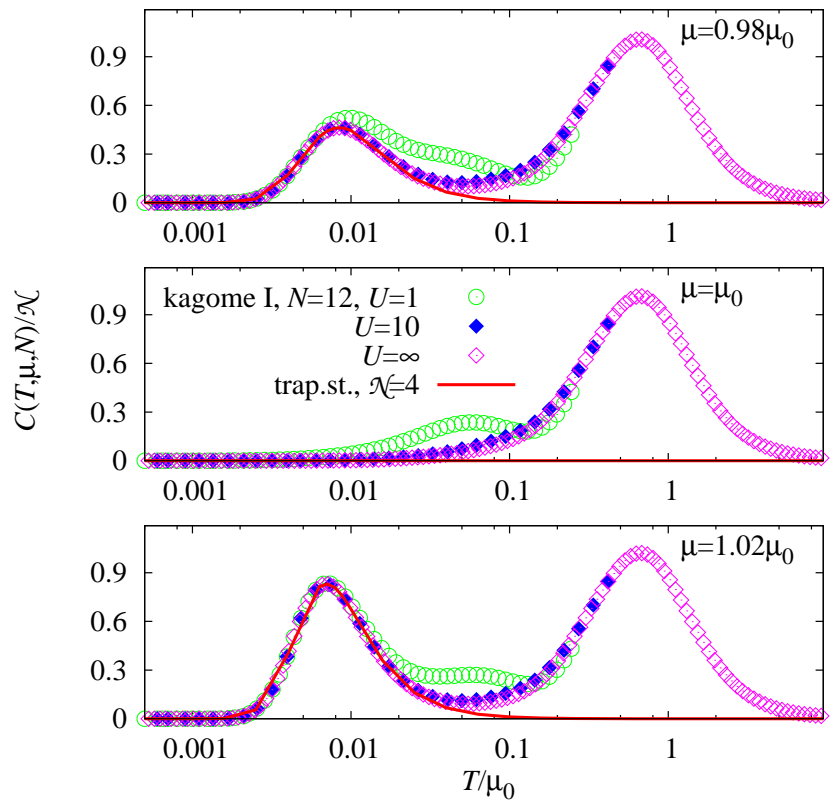

FIG. 8: (Color online) $C(T, \mu, N) / \mathcal{N}$ versus $T / \mu_{0}$ for the kagomé chain I with $N=12$ sites and $t=1$ for different values of $U[U=1$ (empty circles), $U=10$ (filled diamonds), $U \rightarrow \infty$ (empty diamonds)]. The results for finite $U$ were obtained taking into account the sectors with up to 8 electrons. We also show the result which follows from Eq. 5.5 for $\mathcal{N}=4$ (lines).

I with $N=12$ sites and show $C(T, \mu, N) / \mathcal{N}$ versus $T / \mu_{0}$ for $U=1,10, \infty$ (see Fig. 8). For finite $U$ the calculation of thermodynamic quantities becomes very time consuming and therefore we report the contribution of the subspaces with up to a certain number of electrons which is less than $2 N$ thus restricting these data to not too high temperatures. As can be seen from these data, the features at sufficiently low temperature do not depend on the value of $U>0$. Note, however, that at $U=1$ there are some visible corrections down to temperatures $T / \mu_{0}=\mathcal{O}\left(10^{-2}\right)$.

From the experimental point of view it is important to discuss the stability of the features determined by localized electron states with respect to small deviations from the ideal lattice geometry. Then the conditions for the existence of flat bands are violated, and, as a result, the exact degeneracy of the ground states in the subspaces with $n=1, \ldots, n_{\max }$ electrons is lifted. However, we are still faced with a set of a large number of lowlying energy levels which may dominate low-temperature thermodynamics as $\mu$ is around $\mu_{0}$. Deviations from ideal geometry for the sawtooth chain (i.e., when $t^{\prime}$ slightly departs from the value $\sqrt{2} t$ ) has been discussed already in Refs. 32b and 37b. Below we consider the kagomé chain I assuming the hoppings along legs to be slightly different from the hoppings along diamonds. Specifically, we put $t_{d}=1$ along the diamonds and $t_{L}=1.01$ along the two

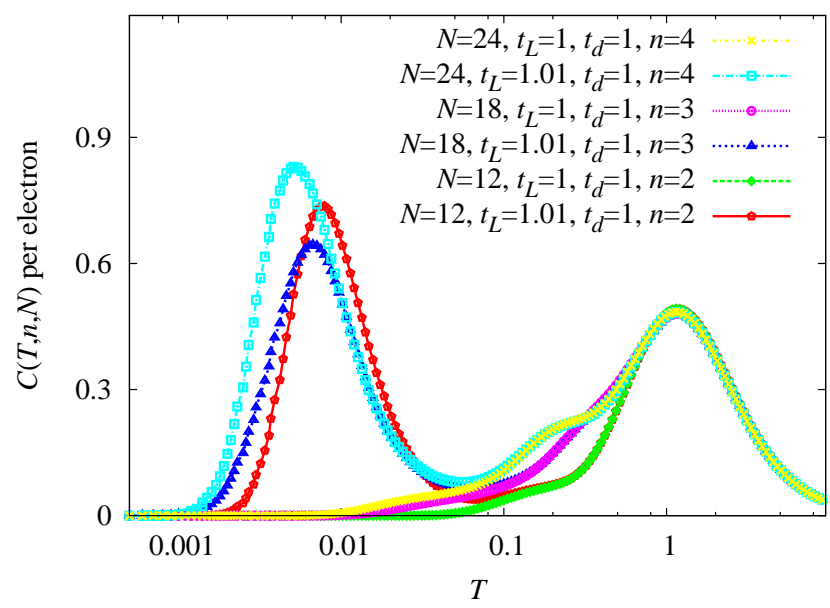

FIG. 9: (Color online) Canonical specific heat $C(T, n, N) / n$ versus $T$ for ideal $\left(t_{d}=t_{L}=1\right)$ and distorted $\left(t_{d}=1\right.$ and $\left.t_{L}=1.01\right)$ kagomé chains I with $n=N / 6$ electrons; $U \rightarrow \infty$.

legs. In Fig. 9 we report the results for the temperature dependence of the canonical specific heat $C(T, n, N) / n$ for $n=\mathcal{N} / 2=N / 6$ electrons. For the ideal lattice $C(T, n, N)=0$ if $n \leq n_{\max }$ in the low-temperature regime (Fig. 9). A deviation from exact degeneracy $\left(t_{d}=1 \neq t_{L}=1.01\right)$ produces a low-temperature peak (Fig. 9). This peak indicates a separation of two energy scales, one is related to the manifold of low-lying trapped states, and the other one to the ordinary extended states. The position of this extra peak depends on $t_{d}-t_{L}$, for instance for $t_{d}=1 \neq t_{L}=1.1$ and $N=24$ the peak is at $T=0.045$. Although there are finite-size effects in the height and the position of the peak its existence is not questioned. We notice that the temperature dependence of the specific heat $C(T, n, N) / n$ is an experimentally accessible quantity and its well-pronounced low-temperature features conditioned by localized electron states for small deviations from ideal lattice geometry may increase chances to observe localized electron state effects.

\section{MAGNETIC PROPERTIES}

The study of ground-state magnetic properties of the Hubbard model (1.1) on the considered lattices is of great interest and has been discussed since the early 1990s. Thus, the sawtooth chain belongs to the one-dimensional version of Tasaki's lattice. H. Tasaki proved that the ground state of the sawtooth-chain Hubbard model is ferromagnetic and unique [up to the trivial SU(2) degeneracy] if the number of electrons $n=N / 2$ (saturated ground-state ferromagnetism), see Ref. 5a. Later on, numerical studies of sawtooth chains of up to $N=12$ sites by Y. Watanabe and S. Miyashita $\frac{19}{}$ revealed groundstate ferromagnetism (saturated and nonsaturated) for 
other values of $n$. Moreover, it was shown in Ref. 11 that within the localized electron picture the model exhibits full polarization in the ground state for $n=N / 2-1$ electrons (only one-cluster states constitute the set of ground states) and $60 \%$ of the full polarization in the ground state for $n=N / 2-2$ electrons if $N \rightarrow \infty$.

The two considered kagomé chains are line graphs. This has been pointed out explicitly for the kagomé chain $\mathrm{I}, \stackrel{34}{=}$ where this connection has been known for a while,$\underline{33}$ albeit using a different terminology. According to a general theory elaborated by A. Mielke the repulsive Hubbard model on these lattices should have ferromagnetic ground states for the number of electrons $n \leq N / 3$ [periodic odd- $\mathcal{N}$ kagomé chain I (nonbipartite parent graph)], $n \leq N / 3+1$ [periodic even- $\mathcal{N}$ kagomé chain I (bipartite parent graph)], or $n \leq N / 5+1$ [periodic kagomé chain II (bipartite parent graph)] (Theorem of Ref. 4a or Theorem 1 of Ref. [ $\mathrm{b}$ b). Moreover, according to A. Mielke the ferromagnetic ground state is unique apart from degeneracy due to $\mathrm{SU}(2)$ invariance (i.e., saturated ground-state ferromagnetism), if $n=N / 3$ (periodic odd- $\mathcal{N}$ kagomé chain I), $n=N / 3+1$ (periodic even- $\mathcal{N}$ kagomé chain I), or $n=N / 5+1$ (periodic kagomé chain II) (Theorem 2 of Ref. $4 \mathrm{~b})$.

To reveal the ferromagnetic ground states we consider the operator

$$
\frac{S^{2}}{\mathcal{N}^{2}}=\frac{\frac{1}{2}\left(S^{+} S^{-}+S^{-} S^{+}\right)+S^{z 2}}{\mathcal{N}^{2}}
$$

with the operators $S^{\alpha}$ defined in (4.2). The average at $T=0,\left\langle\boldsymbol{S}^{2}\right\rangle_{n} / \mathcal{N}^{2}$ is given by the equal-weight average over all degenerate ground states for the given number of electrons $n$. It satisfies

$$
0 \leq \frac{\left\langle\boldsymbol{S}^{2}\right\rangle_{n}}{\mathcal{N}^{2}} \leq \frac{S_{\max }\left(S_{\max }+1\right)}{\mathcal{N}^{2}}, \quad S_{\max }=\frac{n}{2}
$$

If $\left\langle\boldsymbol{S}^{2}\right\rangle_{n} / \mathcal{N}^{2}$ achieves its maximal value the ground state in the subspace with $n$ electrons is the saturated ferromagnetic ground state. If $\left\langle\boldsymbol{S}^{2}\right\rangle_{n} / \mathcal{N}^{2}$ has a nonzero value which is less than the maximal value the ground state in the subspace with $n$ electrons contains ferromagnetic ones and is a nonsaturated ferromagnetic ground state. To examine ground-state magnetism - in particular the existence of ferromagnetism - for thermodynamically large systems one has to consider the limit $\mathcal{N} \rightarrow \infty$, $n \rightarrow \infty$ preserving $n / \mathcal{N}=$ const.

Below we use the constructed many-electron ground states for $n=1, \ldots, n_{\max }$ to discuss systematically ground-state ferromagnetism at electron densities $n \leq$ $n_{\text {max }}$. We complete our analytical arguments by numerics for finite systems. Our results are consistent with general theorems of Tasaki and Mielke for $n=n_{\max }$ and go beyond considering $n<n_{\max }$. Moreover, we also report numerics for higher electron densities $n>n_{\max }$.

We start with a brief overview of our findings: (i) All ground states are fully polarized (saturated ferromagnetism) for $n=\mathcal{N}$ and $n=\mathcal{N}-1$ (all chains) and, in addition, for $n=\mathcal{N}+1$ for the kagomé chain II and the even- $\mathcal{N}$ kagomé chain I. (ii) For smaller $n<\mathcal{N}-1$ the ground-state manifold contains fully and partially polarized as well as paramagnetic states. Thus we have $\left\langle\boldsymbol{S}^{2}\right\rangle_{n}<S_{\max }\left(S_{\max }+1\right)$. The magnetic polarization $\left\langle\boldsymbol{S}^{2}\right\rangle_{n}<S_{\max }\left(S_{\max }+1\right)$ decays monotonically with increasing $\mathcal{N}-n, n<\mathcal{N}$, see Figs. 10 and 11. For large $\mathcal{N}$ the decay becomes very rapid. (iii) While for finite systems there is a finite region of electron density $n / \mathcal{N}$ where ground-state ferromagnetism exists, this region shrinks to one parameter point $n / \mathcal{N}=1$ for $\mathcal{N} \rightarrow \infty$. (iv) For $n / \mathcal{N}<1$ the system shows Curie-like behavior with a uniform zero-field magnetic susceptibility $\chi \propto T^{-1}$.

In what follows, we first discuss separately the case of the systems with hard-dimer ground states only (see Sec.VIA and the case of the systems with ground states which also involve two-leg states (see Sec. VIB). In Sec. VIC we report exact diagonalization data for finite systems at higher electron densities $n>n_{\max }$. We complete our discussion considering the low-temperature behavior of the uniform zero-field magnetic susceptibility for $n \leq n_{\max }$ in Sec. VID.

\section{A. Hard-dimer ground states and ground-state magnetism}

To calculate $\left\langle\boldsymbol{S}^{2}\right\rangle_{n}$ we may further elaborate the $3 \times 3$ transfer-matrix technique. First, we use $\mathrm{SU}(2)$ invariance of the Hubbard model (1.1) to write $\left\langle\boldsymbol{S}^{2}\right\rangle_{n}=$ $3\left\langle S^{z 2}\right\rangle_{n}$. Second, we notice that the average over all degenerate ground states for a given number of electrons $n$ is $\left\langle S^{z^{2}}\right\rangle_{n}=\mathcal{N} \sum_{j=0}^{\mathcal{N}-1}\left\langle S_{0}^{z} S_{j}^{z}\right\rangle_{n}$, where $S_{j}^{z}$ is the $z$-component of the spin operator of the trap $j$. The operator $S_{j}^{z}$ acting on the hard-dimer ground states yields 0 (empty trap), 1/2 (occupied trap with spin-up electron), $-1 / 2$ (occupied trap with spin-down electron). Next with the help of the $3 \times 3$ transfer matrix we find the grandcanonical $z z$ correlation function $\left\langle S_{0}^{z} S_{j}^{z}\right\rangle_{z}$ (the subscript $z$ denotes the activity) yielding the required canonical $z z$ correlation function $\left\langle S_{0}^{z} S_{j}^{z}\right\rangle_{n}$ (see Appendix A). As a result we obtain $\left\langle\boldsymbol{S}^{2}\right\rangle_{n}$ for $n=1, \ldots, \mathcal{N}-1$. We recall that $\left\langle\boldsymbol{S}^{2}\right\rangle_{\mathcal{N}}=(\mathcal{N} / 2)(\mathcal{N} / 2+1)$. An alternative computation based on a hard-dimer mapping (see Appendix A) yields the same results for $\left\langle\boldsymbol{S}^{2}\right\rangle_{n}, n=1, \ldots, \mathcal{N}$.

Using the transfer-matrix method we have calculated $\left\langle\boldsymbol{S}^{2}\right\rangle_{n} / \mathcal{N}^{2}, n=1, \ldots, \mathcal{N}$ for systems of up to $\mathcal{N}=256$, see Fig. 10. We obviously have $\left\langle\boldsymbol{S}^{2}\right\rangle_{n}=S_{\max }\left(S_{\max }+1\right)$ for $n=\mathcal{N}$ and $\mathcal{N}-1$ independently of the system size as predicted above. From the data shown in Fig. 10 we see clearly that $\left\langle\boldsymbol{S}^{2}\right\rangle_{n} / \mathcal{N}^{2}$ becomes smaller for any fixed $n / \mathcal{N}, 0<n / \mathcal{N}<1$, as $\mathcal{N}$ increases (compare the curves for $\mathcal{N}=8$ and $\mathcal{N}=256$ ). More precisely, the curves shown in Fig. 10 suggest $\left\langle\boldsymbol{S}^{2}\right\rangle_{n} / \mathcal{N}^{2} \rightarrow 0$ for $\mathcal{N} \rightarrow \infty$ at $n / \mathcal{N}=$ const. Note that for saturated ferromagnetic ground states $\left\langle\boldsymbol{S}^{2}\right\rangle_{n} / \mathcal{N}^{2}$ would equal $(n / \mathcal{N})^{2} / 4$ in the thermodynamic limit $\mathcal{N} \rightarrow \infty$. In Fig. 10, one observes increasing differences between the 


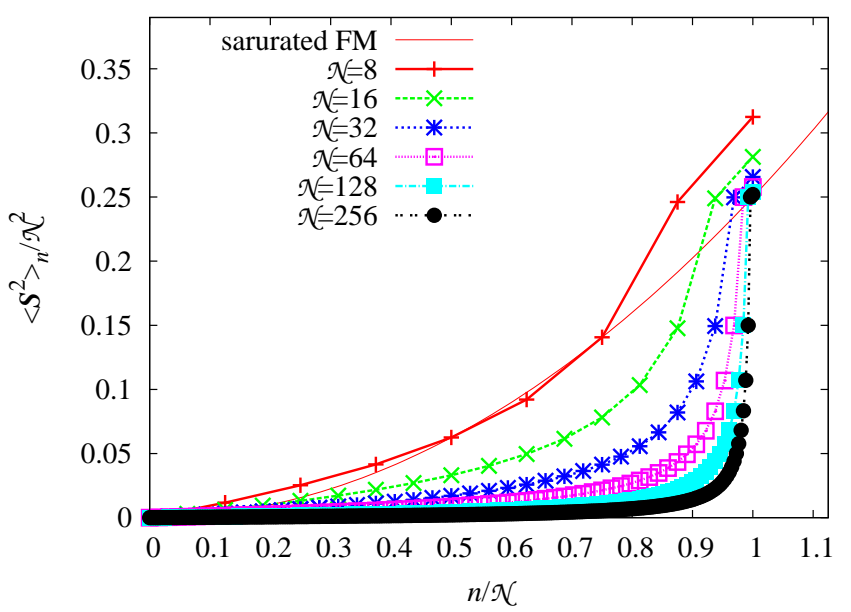

FIG. 10: (Color online) Average ground-state magnetic moment of the sawtooth-Hubbard chain: $\left\langle\boldsymbol{S}^{2}\right\rangle_{n} / \mathcal{N}^{2}$ versus $n / \mathcal{N}$ for $\mathcal{N}=8,16,32,64,128,256$. For saturated ferromagnetic ground states $\left\langle\boldsymbol{S}^{2}\right\rangle_{n} / \mathcal{N}^{2}$ would equal $(n / \mathcal{N})^{2} / 4$ (thin line) in the thermodynamic limit $\mathcal{N} \rightarrow \infty$.

TABLE I: Ground-state $\left\langle\boldsymbol{S}^{2}\right\rangle_{n}$ for $n=1, \ldots, \mathcal{N}$ electrons for the sawtooth-Hubbard chain.

\begin{tabular}{|l||c|c|c|c|c|c|c|c|}
\hline & $n=1$ & $n=2$ & $n=3$ & $n=4$ & $n=5$ & $n=6$ & $n=7$ & $n=8$ \\
\hline \hline $\mathcal{N}=2$ & $\frac{3}{4}$ & 2 & - & - & - & - & - & - \\
\hline $\mathcal{N}=3$ & $\frac{3}{4}$ & 2 & $\frac{15}{4}$ & - & - & - & - & - \\
\hline $\mathcal{N}=4$ & $\frac{3}{4}$ & $\frac{9}{5}$ & $\frac{15}{4}$ & 6 & - & - & - & - \\
\hline $\mathcal{N}=5$ & $\frac{3}{4}$ & $\frac{12}{7}$ & $\frac{63}{20}$ & 6 & $\frac{35}{4}$ & - & - & - \\
\hline $\mathcal{N}=6$ & $\frac{3}{4}$ & $\frac{5}{3}$ & $\frac{81}{28}$ & $\frac{24}{5}$ & $\frac{35}{4}$ & 12 & - & - \\
\hline $\mathcal{N}=7$ & $\frac{3}{4}$ & $\frac{18}{11}$ & $\frac{11}{4}$ & $\frac{30}{7}$ & $\frac{27}{4}$ & 12 & $\frac{63}{4}$ & - \\
\hline $\mathcal{N}=8$ & $\frac{3}{4}$ & $\frac{21}{13}$ & $\frac{117}{44}$ & 4 & $\frac{165}{28}$ & 9 & $\frac{63}{4}$ & 20 \\
\hline
\end{tabular}

curve $(n / \mathcal{N})^{2} / 4$ and the data for $\left\langle\boldsymbol{S}^{2}\right\rangle_{n} / \mathcal{N}^{2}$ which approaches zero for $n / \mathcal{N}<1$. We have performed a finitesize extrapolation of $\left\langle\boldsymbol{S}^{2}\right\rangle_{n} / \mathcal{N}^{2}$ for several fixed electron densities $n / \mathcal{N}=1 / 2,3 / 4,7 / 8$ to estimate the corresponding values for $\mathcal{N} \rightarrow \infty$, and we find, indeed, $\lim _{\mathcal{N} \rightarrow \infty}\left\langle\boldsymbol{S}^{2}\right\rangle_{n} / \mathcal{N}^{2}=0$ for those values of $n / \mathcal{N}$.

In a next step we will give analytical predictions for $\left\langle\boldsymbol{S}^{2}\right\rangle_{n} / \mathcal{N}^{2}$. We first collect the values for $\left\langle\boldsymbol{S}^{2}\right\rangle_{n}$ for sys-

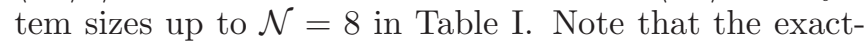
diagonalization data for $\left\langle\boldsymbol{S}^{2}\right\rangle_{n}$ for the sawtooth chain confirm the values given in Table —. By inspecting the

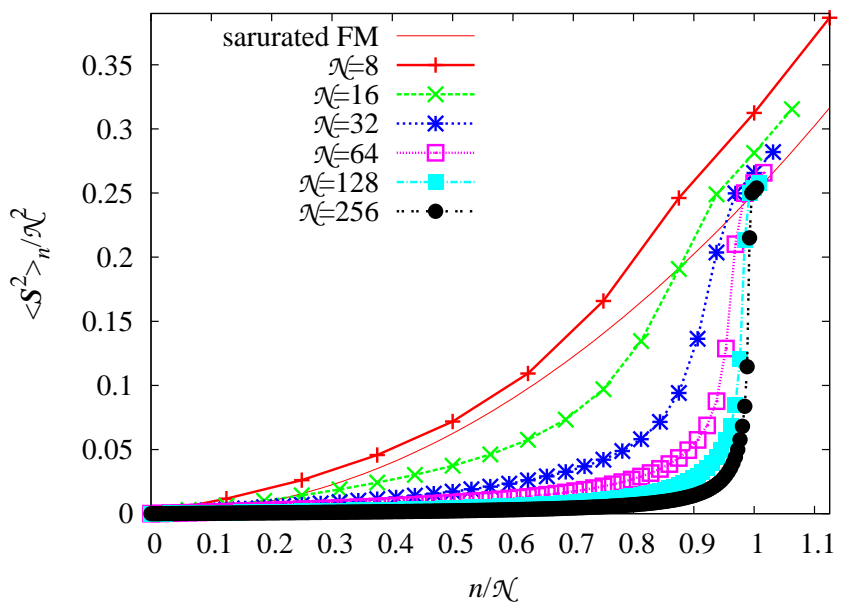

FIG. 11: (Color online) Average ground-state magnetic moment of the kagomé-Hubbard chains: $\left\langle\boldsymbol{S}^{2}\right\rangle_{n} / \mathcal{N}^{2}$ versus $n / \mathcal{N}$ for $\mathcal{N}=8,16,32,64,128,256$. For saturated ferromagnetic ground states $\left\langle\boldsymbol{S}^{2}\right\rangle_{n} / \mathcal{N}^{2}$ would equal $(n / \mathcal{N})^{2} / 4$ (thin line) in the thermodynamic limit $\mathcal{N} \rightarrow \infty$.

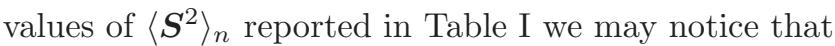

$$
\begin{aligned}
\left\langle\boldsymbol{S}^{2}\right\rangle_{2} & =\frac{6+3(\mathcal{N}-3)}{3+2(\mathcal{N}-3)} \stackrel{\mathcal{N} \rightarrow \infty}{\longrightarrow} \frac{3}{2}, \\
\left\langle\boldsymbol{S}^{2}\right\rangle_{3} & =\frac{45+18(\mathcal{N}-4)}{12+8(\mathcal{N}-4)} \stackrel{\mathcal{N} \rightarrow \infty}{\longrightarrow} \frac{9}{4}, \\
\left\langle\boldsymbol{S}^{2}\right\rangle_{4} & =\frac{54+18(\mathcal{N}-5)}{9+6(\mathcal{N}-5)} \stackrel{\mathcal{N} \rightarrow \infty}{\longrightarrow} 3, \\
\left\langle\boldsymbol{S}^{2}\right\rangle_{5} & =\frac{210+60(\mathcal{N}-6)}{24+16(\mathcal{N}-6)} \stackrel{\mathcal{N} \rightarrow \infty}{\longrightarrow} \frac{15}{4} .
\end{aligned}
$$

This leads to a guess for the thermodynamic limit:

$$
\lim _{\mathcal{N} \rightarrow \infty}\left\langle\boldsymbol{S}^{2}\right\rangle_{n}=\frac{3}{4} n, \quad \frac{n}{\mathcal{N}}<1 .
$$

This result is in accordance with the numerical results presented in Fig. 10, and gives again evidence for a paramagnetic ground state in the infinitely large system.

Summarizing the above analysis we conclude that there is no finite range of ground-state ferromagnetism for electron densities $n / \mathcal{N}<1$ as $\mathcal{N} \rightarrow \infty$.

\section{B. Ground states involving two-leg states and ground-state magnetism}

We turn now to the periodic kagomé chain I with an even number of cells $\mathcal{N}$ and the kagomé chain II for the number of electrons $n \leq \mathcal{N}+1$. As was explained in Sec. IV the states involving two-leg states increase the ground-state degeneracy for $n=1, \ldots, \mathcal{N}$ by the number $L_{\mathcal{N}}(n)$ [see Eq. (4.7)]. All these additional states [except the one state for $n=2$, see Eq. (4.6)] are fully polarized, 
i.e., $\boldsymbol{S}^{2}=(n / 2)(n / 2+1)$. Therefore, for the average value we have $\left\langle\boldsymbol{S}^{2}\right\rangle_{n} \geq\left.\left\langle\boldsymbol{S}^{2}\right\rangle_{n}\right|_{D}$, where $\left.\left\langle\boldsymbol{S}^{2}\right\rangle_{n}\right|_{D}$ is the corresponding value considering hard-dimer states only (i.e., it is that value considered in Sec. VIA see, e.g., Table 【). Recalling that $D_{\mathcal{N}}(n)$ is the number of harddimer ground states one finds

$$
\begin{gathered}
\left\langle\boldsymbol{S}^{2}\right\rangle_{n}=\left.\frac{D_{\mathcal{N}}(n)}{D_{\mathcal{N}}(n)+L_{\mathcal{N}}(n)}\left\langle\boldsymbol{S}^{2}\right\rangle_{n}\right|_{D} \\
+\frac{L_{\mathcal{N}}(n)-\delta_{n, 2}}{D_{\mathcal{N}}(n)+L_{\mathcal{N}}(n)} \frac{n}{2}\left(\frac{n}{2}+1\right) .
\end{gathered}
$$

This formula is valid for $n=1, \ldots, \mathcal{N}-1$. We recall that $\left\langle\boldsymbol{S}^{2}\right\rangle_{\mathcal{N}}=(\mathcal{N} / 2)(\mathcal{N} / 2+1)$ and $\left\langle\boldsymbol{S}^{2}\right\rangle_{\mathcal{N}+1}=$ $[(\mathcal{N}+1) / 2][(\mathcal{N}+1) / 2+1]$. Based on Eq. [6.5) we again can calculate $\left\langle\boldsymbol{S}^{2}\right\rangle_{n}$ for the kagomé chains of up to $\mathcal{N}=256$ cells, see Fig. [11. Noticeable deviations from $\left.\left\langle\boldsymbol{S}^{2}\right\rangle_{n}\right|_{D}$ appear only for small $\mathcal{N}$. Hence the data shown in Fig. 11 give clear evidence that the contribution of the two-leg states becomes irrelevant for large systems. This conclusion is supported by further inspection of Eq. (6.5). Using Eq. (6.4) to replace $\left.\left\langle\boldsymbol{S}^{2}\right\rangle_{n}\right|_{D}$ in the limit $\mathcal{N} \rightarrow \infty$ we can write Eq. 6.5.5) for large $\mathcal{N}$ as

$$
\begin{array}{r}
\lim _{\mathcal{N} \rightarrow \infty}\left\langle S^{2}\right\rangle_{n}=R_{n} \frac{3}{4} n+\left(1-R_{n}\right) \frac{n}{2}\left(\frac{n}{2}-1\right), \\
R_{n}=\lim _{\mathcal{N} \rightarrow \infty} \frac{D_{\mathcal{N}}(n)}{D_{\mathcal{N}}(n)+L_{\mathcal{N}}(n)} .
\end{array}
$$

According to Eqs. (A3), (A44), (5.3), and (5.5) the quantity $D_{\mathcal{N}}(n)$ is proportional to the $n$th derivative of $\xi_{+}^{\mathcal{N}}+\xi_{-}^{\mathcal{N}}+\xi_{3}^{\mathcal{N}}$ with respect to $z$ at $z=0$, whereas $L_{\mathcal{N}}(n)$ is proportional to the $n$th derivative of $\xi_{4}^{\mathcal{N}}+\xi_{5}^{\mathcal{N}}+\xi_{6}^{\mathcal{N}}$ with respect to $z$ at $z=0$. Evaluating derivatives at $z=0$ we find that for $\mathcal{N} \rightarrow \infty$ the ratio $L_{\mathcal{N}}(n) / D_{\mathcal{N}}(n) \propto 1 / \mathcal{N}$ and $\lim _{\mathcal{N} \rightarrow \infty} L_{\mathcal{N}}(n) / D_{\mathcal{N}}(n)=0$. Evidently, $R_{n}=1$ in Eq. (6.6) implies that Eq. (6.4) is valid again, i.e., the ground state is paramagnetic for the infinitely large system.

\section{Results for $n>n_{\max }$}

The flat-band ferromagnets discussed above may exhibit ferromagnetic ground-state ordering even for electron numbers $n>n_{\max }$. However, this ground-state ferromagnetism occurs only for sufficiently large $U$ and therefore is definitely different from the true flat-band ferromagnetism which emerges for any arbitrary small $U$.

The occurrence of a saturated ferromagnetic ground state can be well understood for the sawtooth chain with $n=n_{\max }+1, n_{\max }=\mathcal{N}$. For $U=0$ the sawtooth chain has two single-electron bands separated by the energy gap $\Delta_{1}=\varepsilon_{2}(\pi)-\varepsilon_{1}=2 t$, see Eq. (3.1). If $U$ is small (in comparison with, e.g., $\Delta_{1}$ ) the ground state in the subspace with $n=\mathcal{N}+1$ electrons is a complicated many-body state. However, if $U$ is sufficiently large $U>U_{c}(\mathcal{N}+1)$ (and in particular in the limit $U \rightarrow \infty$ ) it might be energetically favorable to avoid Hubbard repulsion. This can be realized by occupying all trapping cells (V-valleys) with $\mathcal{N}$, say, spin-up electrons and putting the one remaining electron also with $\sigma=\uparrow$ into the next (dispersive) band. Indeed, it is easy to show that for the periodic even- $\mathcal{N}$ sawtooth chain such a state

$$
\left|\varphi_{\mathcal{N}+1}\right\rangle=\alpha_{2, \pi, \uparrow}^{\dagger} l_{0, \uparrow}^{\dagger} l_{2, \uparrow}^{\dagger} \ldots l_{N-2, \uparrow}^{\dagger}|0\rangle
$$

is a true eigenstate with the energy $\mathcal{N} \varepsilon_{1}+\varepsilon_{2}(\pi)$. The trapping-cell operators $l_{2 j, \uparrow}^{\dagger}$ are defined in Eq. (3.2) and $\alpha_{2, \pi, \uparrow}^{\dagger}$ creates an electron in the dispersive band with $\kappa=$ $\pi$ and $\sigma=\uparrow$. Other states belonging to a spin- $[(\mathcal{N}+$ 1)/2] $\mathrm{SU}(2)$ multiplet can be obtained by applying $S^{-}$ to the state (6.7). This kind of saturated ground-state ferromagnetism for the sawtooth chain was first found numerically by Y. Watanabe and S. Miyashita ${ }^{19}$ (see also Refs. 7, 12).

We may expect such fully polarized ferromagnetic ground states for sufficiently large $U$ for further electron numbers $n_{\max }<n<N$, where $n=N-1$ is that electron number where the well-known Nagaoka theorem ${ }^{53}$ holds. Using Lanczos exact diagonalization of finite systems we have investigated this question for the sawtooth and the kagomé chains I and II. We list our numerical findings for $U \rightarrow \infty$ in Table II. Indeed, fully polarized ferromagnetic ground states exist for various electron numbers $n$ in the range $n_{\max }<n<N$. For other values not listed in Table II the ground state is either partially polarized, i.e., $0<\left\langle\boldsymbol{S}^{2}\right\rangle_{n}<S_{\max }\left(S_{\max }+1\right)$, or it is a singlet, i.e., $\left\langle\boldsymbol{S}^{2}\right\rangle_{n}=0$, with spiral structure, see also Ref. 19. However, a detailed discussion of this issue goes beyond the scope of the present paper.

\section{Low-temperature behavior of the magnetic susceptibility}

We complete our discussion of the magnetic properties with a brief consideration of the low-temperature behavior of the uniform zero-field magnetic susceptibility $\chi$. Using the standard arguments for deriving the uniform zero-field Langevin susceptibility we may write the trapped-state contribution to $\chi$ as

$$
\chi(T, n, N)=\frac{\left\langle\boldsymbol{S}^{2}\right\rangle_{n}}{3 T}
$$

with $\left\langle\boldsymbol{S}^{2}\right\rangle_{n}$ calculated in Secs. VIA and VIB. Thus we may expect a Curie-like behavior of the susceptibility of the considered Hubbard chains at low temperatures in case of paramagnetic ground states. In the thermodynamic limit we have $\lim _{\mathcal{N} \rightarrow \infty}\left\langle\boldsymbol{S}^{2}\right\rangle_{n}=3 n / 4$ in the paramagnetic region $(n / \mathcal{N}<1)$, see Eq. (6.4). Therefore the Curie constant is $n / 4$, which corresponds to a system of $n$ independent spins $1 / 2$. Note, however, that in case of a ferromagnetic ground state the low-temperature dependence of $\chi$ is expected to be different. Thus for 
TABLE II: Electron numbers $n_{\max }<n<N$ for which saturated ground-state ferromagnetism in the limit $U \rightarrow \infty$ exists. Note that for $N>20$ the size of the Hamiltonian matrix becomes very large. Therefore, for $N>20$ only a few sectors $n>n_{\text {max }}$ are accessible by numerical calculation. In particular, for $N=24$ we can examine only the sectors with up to $n=16$ electrons and for $N=30$ with up to $n=10$ electrons. Note further that for $N=40$ and $N=50$ we cannot reach the sectors with $n>n_{\text {max }}$.

\begin{tabular}{|c||c|c|c|c|}
\hline chain & $\mathcal{N}=4$ & $\mathcal{N}=6$ & $\mathcal{N}=8$ & $\mathcal{N}=10$ \\
\hline \hline sawtooth & $N=8: n=5,7$ & $N=12: n=7,9,11$ & $N=16: n=9,11,13,15$ & $N=20: n=11,13,15,17,19$ \\
\hline kagomé I & $N=12: n=8,11$ & $N=18: n=9,10,14,15,17$ & $N=24: n=11,-$ & $N=30:-$ \\
\hline kagomé II & $N=20: n=7,8,10,13,17,19$ & $N=30: n=9,10,-$ & $N=40:-$ & $N=50:-$ \\
\hline
\end{tabular}

the spin-1/2 ferromagnetic Heisenberg chain the Bethe ansatz yields $\chi \propto T^{-2}$ (see Ref. 54) which holds also for weakly frustrated chains $\underline{55}$

Again we have confirmed Eq. (6.8) by numerical calculations for finite systems. As an example some results for the sawtooth chain of two different lengths are presented in Fig. 12 for $t^{\prime}=\sqrt{2}$ and $t^{\prime}=1$. From Fig. 12 it is obvious that for the sawtooth chain with a flat band, i.e., $t^{\prime}=\sqrt{2}$, Eq. (6.8) holds at low temperatures. Moreover, the obtained Curie constants imply $\left\langle\boldsymbol{S}^{2}\right\rangle_{4}=4$ for $\mathcal{N}=8$ and $\left\langle\boldsymbol{S}^{2}\right\rangle_{4}=60 / 17$ for $\mathcal{N}=12$ in agreement with calculations of Sec. VIA The temperature region where this relation is valid increases with growing $U$. In case of a dispersive lowest band, i.e., $t^{\prime}=1$, the quantity $\left\langle\boldsymbol{S}^{2}\right\rangle_{n}$ depends on $U$ and $n$. Indeed, it has been observed previously $\frac{38}{3}$ that in the sawtooth chain with $t^{\prime}=t$ for quarter filling and less than quarter filling the Coulomb repulsion $U$ may drive transitions from singlet ground states present at small values of $U$ to ferromagnetic ground states present at large values of $U$. For the values of $U$ and $n$ considered in Fig. 12 we have singlet ground states, i.e., $\left\langle\boldsymbol{S}^{2}\right\rangle_{n}=0$, for $t^{\prime}=1$. Hence, contrary to the flat-band case we have $T \chi(T, n, N)=0$ as $T \rightarrow 0$.

\section{RELATION TO THE $X X Z$ MODEL}

Finally we want discuss the relation between the exact many-electron states considered in this paper and the localized magnon states found for the $X X Z$ Heisenberg antiferromagnet $\stackrel{26,29,30}{\underline{.}}$

First we notice that the localized magnon states for the $X X Z$ Heisenberg antiferromagnet on all three lattices can be also mapped onto the model of hard dimers on an auxiliary simple chain. However, in contradistinction to the electron model, the localized magnons cannot sit in neighboring traps and there is only one possibility to occupy a trap. Moreover, for the kagomé chains the simultaneous occupation of a leg and a diamond or hexagon trapping cell by magnons is not allowed. Hence, we are faced with an example where the Pauli principle leads to less constraints in comparison to hard-core bosonic systems. As a result, the number of states for the $X X Z$ model is given by hard dimers on a chain of only $\mathcal{N}$ sites instead of the $2 \mathcal{N}$ sites as is the case for the Hubbard model $\underline{29,30}$

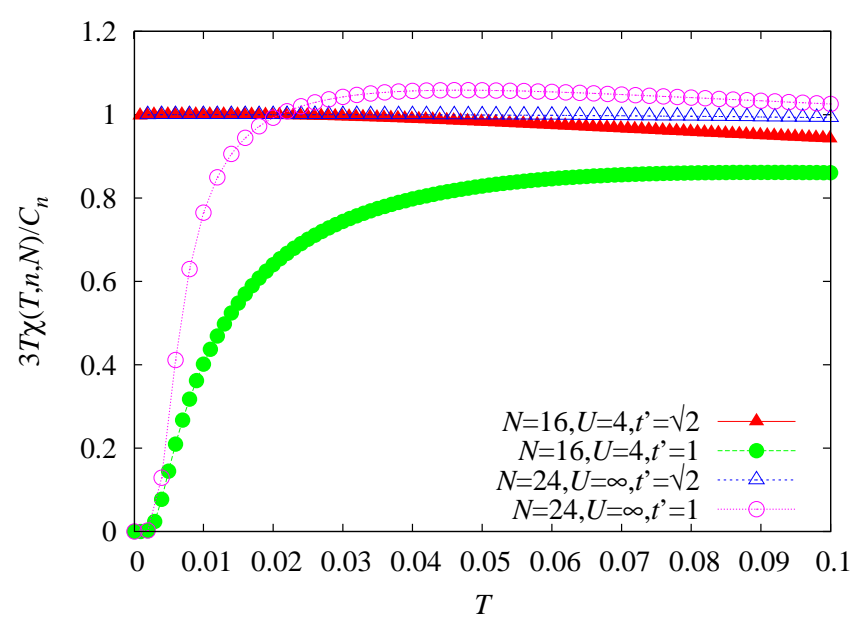

FIG. 12: (Color online) Uniform magnetic susceptibility $3 T \chi(T, n, N) / C_{n}$ for the sawtooth chain $(t=1)$ with $n=4$ electrons and $N=16$ sites with $U=4$ (filled triangles and circles) as well as $N=24$ sites with $U \rightarrow \infty$ (empty triangles and circles). Triangles correspond to $t^{\prime}=\sqrt{2}$ and circles correspond to $t^{\prime}=1$. Here we use for the normalization of the vertical axis $C_{n}=4$ for $\mathcal{N}=8$ and $C_{n}=60 / 17$ for $\mathcal{N}=12$.

Further differences can be emphasized between the localized magnons and the localized electrons for these three chains. First, in the subspace with $n=\mathcal{N} / 2$ magnons the ground-state degeneracy equals 2 whereas in the corresponding subspace with $n=\mathcal{N}$ electrons the hard-dimer ground-state degeneracy equals $\mathcal{N}+1$. For the antiferromagnetic Heisenberg kagomé chains the extended single-magnon states (two-leg states) also appear, however, because of the stronger constraint only in the subspaces with $n=1$ and $n=2$ magnons. This increases the total ground-state degeneracy at the saturation magnetic field $h=h_{\text {sat }}$ exactly by 2 (compare Ref. 30b). By contrast, for the Hubbard kagomé chains the extended single-electron states (two-leg states) appear in all subspaces with $n=1, \ldots, \mathcal{N}+1$ electrons thus noticeably increasing the ground-state degeneracy at $\mu=\mu_{0}$.

Despite the differences between the fermionic and the hard-core bosonic systems stressed above, the existence of localized states in both systems leads to some common features. For instance, the jump in the number of 
electrons as a function of the chemical potential $\mu$ at $\mu=\mu_{0}$ found for the electron system corresponds to a jump in the magnetization curve as a function of the external magnetic field at the saturation field $h=h_{\text {sat }}$ seen in the $X X Z$ model. Moreover, in both cases the contribution of the localized states to the partition function can be calculated explicitly by a transfer-matrix method, which leads to simple analytical expressions for the lowtemperature behavior of various thermodynamic quantities, such as the entropy or the specific heat.

\section{CONCLUSIONS}

In this paper, we have considered two different types of flat-band ferromagnets, namely, the sawtooth Hubbard chain (Tasaki's model) and two kagomé Hubbard chains (Mielke's models). For these three models we have constructed the complete set of ground states for electron numbers $n \leq n_{\max }$, where $n_{\max }=\mathcal{N}$ (sawtooth, odd- $\mathcal{N}$ kagomé I) or $n_{\max }=\mathcal{N}+1$ (even- $\mathcal{N}$ kagomé I, kagomé II) with $\mathcal{N}=N / 2$ (sawtooth), $\mathcal{N}=N / 3$ (kagomé I), and $\mathcal{N}=N / 5$ (kagomé II). In these ground states the electrons are trapped on restricted areas of the full lattice. Using a transfer-matrix method, see Appendix $\mathrm{A}$ we have calculated the degeneracy of the ground states $g_{\mathcal{N}}(n)$. The ground-state degeneracy grows rapidly with increasing system size, and a finite residual entropy per site remains in the thermodynamic limit. Moreover, we have calculated exactly the contribution of the highly degenerate ground-state manifold to the partition function. The low-temperature thermodynamics around a particular value of the chemical potential $\mu_{0}\left(\mu_{0}=2 t\right.$ for all three models) is dominated by these trapped ground states leading to a low-energy scale separated from the usual energy scale determined by the band width. In the thermodynamic limit $N \rightarrow \infty$ all models exhibit identical thermodynamic behavior in this regime which is analogous to that of classical one-dimensional hard dimers.

The trapped ground states lead also to particular magnetic behavior including ground-state ferromagnetism as well as paramagnetic behavior.

Moreover, with this study we have (i) illuminated relations between Tasaki's and Mielke's flat-band ferromagnets, and (ii) the relations between frustrated quantum Heisenberg antiferromagnets and the Hubbard flat-band ferromagnets. In spite of some similarities in the mathematical description of both correlated quantum lattice systems owing to localized one-particle states, the elaboration of a comprehensive theory for the Hubbard flatband ferromagnet in higher dimensions using some ideas from localized-spin systems remains an unsolved problem and calls for further efforts.

\section{Acknowledgments}

The numerical calculations were performed using J. Schulenburg's spinpack. Financial support of the DFG is gratefully acknowledged (projects Ri615/16-1 and Ri615/18-1 and a Heisenberg fellowship for A.H. under project HO 2325/4-1). O.D. acknowledges the kind hospitality of the MPIPKS-Dresden in 2006 and 2009 and of the University of Magdeburg in the autumn of 2008 and 2009.

\section{APPENDIX A: TRANSFER-MATRIX COUNTING OF LOCALIZED HARD-DIMER ELECTRON STATES}

The ground states of electrons localized on trapping cells (V-valleys, diamonds or hexagons, respectively) for $U>0$ can be counted using a transfer-matrix method. $\underline{49}$ In Ref. 11 we have associated two sites to each trapping cell and used a hard-dimer mapping for this counting. Here we will present a direct solution of the same problem using a $3 \times 3$ transfer matrix.

Recall that the ground states of the considered systems, i.e., the sawtooth chain or the odd- $\mathcal{N}$ kagomé chain I with $\mu$ around $\mu_{0}$, can be obtained by populating the trapping cells with electrons with spin-up and spin-down according to the following rules: 1) Each trap may be empty, occupied by one spin-up electron or occupied by one spin-down electron. That is, for each trap $j=0,1, \ldots, \mathcal{N}-1$ we have three trap states, $s_{j}=0, \uparrow, \downarrow$. A ground state of the chain can be thought of as a certain sequence of the trap states. 2) Moving along the successive traps (the choice of the first trap is totally arbitrary, e.g., we may take $j=0$ ) we must allow only one sequence of two trap states corresponding to the neighboring cells being occupied by differently polarized electrons. This is only a convention for the correct counting of the number of the ground states. (Note, however, that the convention does not work for the number of electrons $n=\mathcal{N}$ yielding only 2 states instead of correct number $\mathcal{N}+1$.) For instance, let us allow the sequence of trap states $s_{j}=\uparrow$, $s_{j+1}=\downarrow$ and forbid the sequence of trap states $s_{j}=\downarrow$, $s_{j+1}=\uparrow$.

These rules can be encoded with a transfer matrix ${ }^{56}$

$$
\begin{aligned}
\mathbf{T} & =\left(\begin{array}{lll}
T(0,0) & T(0, \uparrow) & T(0, \downarrow) \\
T(\uparrow, 0) & T(\uparrow, \uparrow) & T(\uparrow, \downarrow) \\
T(\downarrow, 0) & T(\downarrow, \uparrow) & T(\downarrow, \downarrow)
\end{array}\right) \\
& =\left(\begin{array}{lll}
1 & 1 & 1 \\
z & z & z \\
z & 0 & z
\end{array}\right)
\end{aligned}
$$

with the activity $z=\exp \left(-\varepsilon_{1} / T\right)$. Now we can write down the contribution of all allowed sequences $s_{0}, \ldots, s_{\mathcal{N}-1}$ to the grand-canonical partition function 
$\Xi_{\text {trap }}(z, \mathcal{N})(5.2)$ as follows

$$
\begin{aligned}
\Xi_{\text {trap }}(z, \mathcal{N}) & =\operatorname{Tr} \mathbf{T}^{\mathcal{N}} \\
& =\xi_{+}^{\mathcal{N}}+\xi_{-}^{\mathcal{N}}+\xi_{0}^{\mathcal{N}} \\
\xi_{ \pm} & =\frac{1}{2}+z \pm \sqrt{\frac{1}{4}+z}, \quad \xi_{0}=0 .
\end{aligned}
$$

The hard-dimer computation yields the alternative representation $\Xi_{\text {trap }}(z, \mathcal{N})=\lambda_{+}^{2 \mathcal{N}}+\lambda_{-}^{2 \mathcal{N}}$ where $\lambda_{ \pm}=1 / 2 \pm$ $\sqrt{1 / 4+\exp x}$ are the eigenvalues of a $2 \times 2$ transfer matrix. ${ }^{11}$ Since $\xi_{ \pm}=\lambda_{ \pm}^{2}$ and $\xi_{0}=0$, the two expressions are in fact equivalent.

We turn now to the canonical description. More specifically, we consider $n \leq \mathcal{N}$ trapped states on a (periodic) chain with $\mathcal{N}$ cells. We are interested in the canonical partition function of such a system $\mathcal{Z}(n, \mathcal{N})$ which counts the number of spatial configurations of $n$ trapped electrons. Using the relation between the canonical partition function and the grand-canonical partition function

$$
\Xi_{\text {trap }}(z, \mathcal{N})=\sum_{n=0}^{\mathcal{N}} z^{n} \mathcal{Z}(n, \mathcal{N})
$$

we immediately find that

$$
\mathcal{Z}(n, \mathcal{N})=\left.\frac{1}{n !} \frac{d^{n} \Xi_{\operatorname{trap}}(z, \mathcal{N})}{d z^{n}}\right|_{z=0}
$$

After simple (but becoming tedious as $n$ increases) calculations we get

$$
\begin{aligned}
& \mathcal{Z}(1, \mathcal{N})=2 \mathcal{N} \\
& \mathcal{Z}(2, \mathcal{N})=\mathcal{N}(2 \mathcal{N}-3) \\
& \mathcal{Z}(3, \mathcal{N})=\frac{1}{3 !} 2 \mathcal{N}\left(4 \mathcal{N}^{2}-18 \mathcal{N}+20\right) \\
& \mathcal{Z}(4, \mathcal{N})=\frac{1}{4 !} 2 \mathcal{N}\left(8 \mathcal{N}^{3}-72 \mathcal{N}^{2}+214 \mathcal{N}-210\right) \\
& \mathcal{Z}(5, \mathcal{N})=\frac{1}{5 !} 2 \mathcal{N}\left(16 \mathcal{N}^{4}-240 \mathcal{N}^{3}+1340 \mathcal{N}^{2}-3300 \mathcal{N}+3024\right) \\
& \mathcal{Z}(6, \mathcal{N})=\frac{1}{6 !} 2 \mathcal{N}\left(32 \mathcal{N}^{5}-720 \mathcal{N}^{4}+6440 \mathcal{N}^{3}-28620 \mathcal{N}^{2}+63188 \mathcal{N}-55440\right) \\
& \mathcal{Z}(7, \mathcal{N})=\frac{1}{7 !} 2 \mathcal{N}\left(64 \mathcal{N}^{6}-2016 \mathcal{N}^{5}+26320 \mathcal{N}^{4}-182280 \mathcal{N}^{3}+706216 \mathcal{N}^{2}-1451184 \mathcal{N}+1235520\right) \\
& \mathcal{Z}(8, \mathcal{N})=\frac{1}{8 !} 2 \mathcal{N}\left(128 \mathcal{N}^{7}-5376 \mathcal{N}^{6}+96320 \mathcal{N}^{5}-954240 \mathcal{N}^{4}+5645192 \mathcal{N}^{3}-19941264 \mathcal{N}^{2}+38943000 \mathcal{N}-32432400\right)
\end{aligned}
$$

The data shown in Fig. 3 are based on Eq. (A5).

Let us calculate the (not normalized) grand-canonical correlation function $\left\langle S_{0}^{z} S_{j}^{z}\right\rangle_{z}$, where $S_{j}^{z}$ is the $z$ component spin operator of the trap $j$ and the subscript $z$ denotes the activity. Defining a matrix

$$
\mathbf{S}=\left(\begin{array}{ccc}
0 & 0 & 0 \\
0 & \frac{1}{2} & 0 \\
0 & 0 & -\frac{1}{2}
\end{array}\right)
$$

one passes again from the sum over hard-dimer ground states for a fixed number of electrons $n$ and the sum over $n \leq \mathcal{N}$ to the sum over $s_{0}=0, \uparrow, \downarrow, s_{1}=0, \uparrow, \downarrow, \ldots$, $s_{\mathcal{N}-1}=0, \uparrow, \downarrow$. As a result

$$
\left\langle S_{0}^{z} S_{j}^{z}\right\rangle_{z}=\operatorname{Tr}\left(\mathbf{S} \mathbf{T}^{j} \mathbf{S} \mathbf{T}^{\mathcal{N}-j}\right) .
$$

Using a MAPLE code we can easily compute $\left\langle S_{0}^{z} S_{j}^{z}\right\rangle_{z}$ according to Eq. (A7) for sufficiently large systems (up to $\mathcal{N}=256$ ); the resulting expression for $\left\langle S_{0}^{z} S_{j}^{z}\right\rangle_{z}$ is a polynomial with the powers of $z$ from 2 to $\mathcal{N}$.
Turning to the canonical description we use the relation

$$
\left\langle S_{0}^{z} S_{j}^{z}\right\rangle_{z}=\sum_{n=2}^{\mathcal{N}} z^{n} \mathcal{Z}(n, \mathcal{N})\left\langle S_{0}^{z} S_{j}^{z}\right\rangle_{n}
$$

[obviously $\left\langle S_{0}^{z} S_{j}^{z}\right\rangle_{n=0}=\left\langle S_{0}^{z} S_{j}^{z}\right\rangle_{n=1}=0$ do not enter the right-hand side of Eq. (A8)] to derive

$$
\mathcal{Z}(n, \mathcal{N})\left\langle S_{0}^{z} S_{j}^{z}\right\rangle_{n}=\left.\frac{1}{n !} \frac{d^{n}\left\langle S_{0}^{z} S_{j}^{z}\right\rangle_{z}}{d z^{n}}\right|_{z=0} .
$$

Thus the coefficients associated with the corresponding powers of the activity $z$ in the right-hand side in Eq. (A7) yield the required quantities $\left\langle S_{0}^{z} S_{j}^{z}\right\rangle_{n}$.

Finally, we mention that the same results for $\left\langle S^{2}\right\rangle_{n}$ can be obtained within the hard-dimer picture. Since each hard-dimer ground state can be represented in terms of the occupation numbers of hard dimers on a simple chain, i.e., it is enumerated by a set of hard-dimer 
occupation numbers $n_{0}, n_{1}, \ldots, n_{2 \mathcal{N}-1}, n_{j}=0,1$, and $S^{z}=(1 / 2) \sum_{j=0}^{2 \mathcal{N}-1}(-1)^{j} n_{j}$, we have

$$
\left\langle S^{z^{2}}\right\rangle_{n}=\frac{\mathcal{N}}{2} \sum_{q=0}^{2 \mathcal{N}-1}(-1)^{q}\left\langle n_{0} n_{q}\right\rangle_{n},
$$

where $\langle\ldots\rangle_{n}$ in the right-hand side in Eq. A10 stands for the (normalized) average over spatial configurations of $n$ hard dimers on a simple chain of $2 \mathcal{N}$ sites. To find a density-density correlation function at distance $q$ for one-dimensional hard dimers in the canonical ensemble $(n, 2 \mathcal{N})$, it is convenient to calculate first a density-density correlation function at distance $q$ for onedimensional hard dimers in the grand-canonical ensemble $(z, 2 \mathcal{N})$,

$$
\begin{array}{r}
\left\langle n_{0} n_{q}\right\rangle_{z}=\operatorname{Tr}\left(\mathbf{N D}^{q} \mathbf{N D}^{2 \mathcal{N}-q}\right), \\
\mathbf{D}=\left(\begin{array}{cc}
1 & \sqrt{z} \\
\sqrt{z} & 0
\end{array}\right), \quad \mathbf{N}=\left(\begin{array}{ll}
0 & 0 \\
0 & 1
\end{array}\right),
\end{array}
$$

which gives the required $\left\langle n_{0} n_{q}\right\rangle_{n}$ after inverting the relation $\left\langle n_{0} n_{q}\right\rangle_{z}=\sum_{n=2}^{\mathcal{N}} z^{n} \mathcal{Z}(n, \mathcal{N})\left\langle n_{0} n_{q}\right\rangle_{n}$.

\section{APPENDIX B: LINEAR INDEPENDENCE OF TRAPPED ELECTRON STATES}

We wish to clarify whether the set of ground states constructed in Secs. IVA and IVB for given $n \leq n_{\max }$ is linearly independent. An affirmative answer for harddimer states comes from Ref. 48. For the sawtooth chain and the kagomé chains the localized $n$-electron states $(n=1, \ldots, \mathcal{N})$ are linearly independent, which is connected with the fact that for all three lattices there are sites which are unique to each cell (isolated class in the nomenclature of Ref. 48).

We can use the same arguments for the set of singleelectron states which consists of localized states on a diamond/hexagon plus one two-leg state. For this purpose the two-leg state (3.5)/(3.9) in the set of states can be replaced by the state localized along one (e.g., lower) leg only. As a result we again are faced with the case when there are isolated sites [the sites belonging to another (upper) leg] that yields linear independence of the considered set of single-electron states and thus of $n$-electron states $(n=1, \ldots, \mathcal{N}+1) \underline{\underline{48}}$
1 The Hubbard Model - A Reprint Volume, edited by A. Montorsi (World Scientific, Singapore, 1992).

2 E. H. Lieb, in XIth International Congress of Mathematical Physics, Paris, 1994, edited by D. Iagolnitzer (International Press, Boston, 1995), p. 392 arXiv:cond-mat/9311033.

3 H. Tasaki, J. Phys.: Condens. Matter 10, 4353 (1998).

4 A. Mielke, J. Phys. A 24, L73 (1991); A. Mielke, J. Phys. A 24, 3311 (1991); A. Mielke, J. Phys. A 25, 4335 (1992).

${ }^{5}$ H. Tasaki, Phys. Rev. Lett. 69, 1608 (1992); A. Mielke and H. Tasaki, Commun. Math. Phys. 158, 341 (1993).

${ }^{6}$ H. Tasaki, Prog. Theor. Phys. 99, 489 (1998).

7 A. Tanaka and T. Idogaki, J. Phys. Soc. Jpn. 67, 401 (1998).

8 C. D. Batista and B. S. Shastry, Phys. Rev. Lett. 91, 116401 (2003).

9 Z. Gulácsi, A. Kampf, and D. Vollhardt, Phys. Rev. Lett. 99, 026404 (2007); Z. Gulácsi, A. Kampf, and D. Vollhardt, Prog. Theor. Phys. Suppl. 176, 1 (2008); R. Trencsényi, E. Kovács, and Z. Gulácsi, Philos. Mag. 89, 1953 (2009).

10 C. Wu, D. Bergman, L. Balents, and S. Das Sarma, Phys. Rev. Lett. 99, 070401 (2007); C. Wu and S. Das Sarma, Phys. Rev. B 77, 235107 (2008).

11 O. Derzhko, A. Honecker, and J. Richter, Phys. Rev. B 76, 220402(R) (2007).

12 O. Derzhko, A. Honecker, and J. Richter, Phys. Rev. B 79, 054403 (2009).

13 D. Vollhardt, N. Blümer, K. Held, M. Kollar, J. Schlipf, and M. Ulmke, Z. Phys. B 103, 283 (1997).

14 H. Tasaki, Phys. Rev. Lett. 75, 4678 (1995); H. Tasaki, J. Stat. Phys. 84, 535 (1996).

15 A. Mielke, Phys. Rev. Lett. 82, 4312 (1999); A. Mielke, J. Phys. A 32, 8411 (1999).

16 A. Tanaka and H. Ueda, Phys. Rev. Lett. 90, 067204
(2003).

17 T. Sekizawa, J. Phys. A 36, 10451 (2003).

18 A. Tanaka and H. Tasaki, Phys. Rev. Lett. 98, 116402 (2007).

19 Y. Watanabe and S. Miyashita, J. Phys. Soc. Jpn. 66, 2123 (1997); Y. Watanabe and S. Miyashita, J. Phys. Soc. Jpn. 66, 3981 (1997); Y. Watanabe and S. Miyashita, J. Phys. Soc. Jpn. 68, 3086 (1999); R. Arita and H. Aoki, Phys. Rev. B 61, 12261 (2000).

${ }^{20}$ H. Tamura, K. Shiraishi, T. Kimura, and H. Takayanagi, Phys. Rev. B 65, 085324 (2002); M. Ichimura, K. Kusakabe, S. Watanabe, and T. Onogi, Phys. Rev. B 58, 9595 (1998); H. Ishii, T. Nakayama, and J.-i. Inoue, Phys. Rev. B 69, 085325 (2004).

21 S. Nishino, M. Goda, and K. Kusakabe, J. Phys. Soc. Jpn. 72, 2015 (2003); S. Nishino and M. Goda, J. Phys. Soc. Jpn. 74, 393 (2005).

22 H.-H. Lin, T. Hikihara, H.-T. Jeng, B.-L. Huang, C.Y. Mou, and X. Hu, Phys. Rev. B 79, 035405 (2009).

23 R. Arita, Y. Suwa, K. Kuroki, and H. Aoki, Phys. Rev. Lett. 88, 127202 (2002); Y. Suwa, R. Arita, K. Kuroki, and H. Aoki, Phys. Rev. B 68, 174419 (2003); A. Harrison, J. Phys.: Condens. Matter 16, S553 (2004); Y.-Z. Zheng, M.-L. Tong, W. Xue, W.-X. Zhang, X.-M. Chen, F. Grandjean, and G. J. Long, Angew. Chem. Int. Ed. 46, 6076 (2007).

24 D. Jaksch and P. Zoller, Ann. Phys. (N.Y.) 315, 52 (2005).

25 M. Lewenstein, A. Sanpera, V. Ahufinger, B. Damski, A. Sen, and U. Sen, Adv. Phys. 56, 243 (2007).

26 J. Schnack, H.-J. Schmidt, J. Richter, and J. Schulenburg, Eur. Phys. J. B 24, 475 (2001); J. Schulenburg, A. Honecker, J. Schnack, J. Richter, and H.-J. Schmidt, Phys. Rev. Lett. 88, 167207 (2002); J. Richter, J. Schulenburg, A. Honecker, J. Schnack, and H.-J. Schmidt, 
J. Phys.: Condens. Matter 16, S779 (2004); J. Richter, J. Schulenburg, and A. Honecker, in Quantum Magnetism, edited by U. Schollwöck, J. Richter, D. J. J. Farnell, and R. F. Bishop, Lecture Notes in Physics, 645 (Springer, Berlin, 2004), pp. 85-153; J. Richter, J. Schulenburg, A. Honecker, and D. Schmalfuß, Phys. Rev. B 70, 174454 (2004); J. Richter, Fizika Nizkikh Temperatur (Kharkiv) 31, 918 (2005) [Low Temperature Physics 31, 695 (2005)].

27 J. Richter, O. Derzhko, and J. Schulenburg, Phys. Rev. Lett. 93, 107206 (2004); O. Derzhko and J. Richter, Phys. Rev. B 72, 094437 (2005).

${ }^{28}$ M. E. Zhitomirsky and A. Honecker, J. Stat. Mech.: Theor. Exp. P07012 (2004).

29 M. E. Zhitomirsky and H. Tsunetsugu, Phys. Rev. B 70, 100403(R) (2004); M. E. Zhitomirsky and H. Tsunetsugu, Prog. Theor. Phys. Suppl. 160, 361 (2005); M. E. Zhitomirsky and H. Tsunetsugu, Phys. Rev. B 75, 224416 (2007).

30 O. Derzhko and J. Richter, Phys. Rev. B 70, 104415 (2004); O. Derzhko and J. Richter, Eur. Phys. J. B 52, 23 (2006); J. Richter, O. Derzhko, and T. Krokhmalskii, Phys. Rev. B 74, 144430 (2006); O. Derzhko, J. Richter, A. Honecker, and H.-J. Schmidt, Fizika Nizkikh Temperatur (Kharkiv) 33, 982 (2007) [Low Temperature Physics 33, 745 (2007)].

31 J. Schnack, R. Schmidt, and J. Richter, Phys. Rev. B 76, 054413 (2007).

32 A. Honecker and J. Richter, Condensed Matter Physics (L'viv) 8, 813 (2005); J. Richter, O. Derzhko, and A. Honecker, Int. J. Mod. Phys. B 22, 4418 (2008); O. Derzhko, J. Richter, and A. Honecker, J. Phys.: Conf. Ser. 145, 012059 (2009).

33 G. Misguich, D. Serban, and V. Pasquier, Phys. Rev. B 67, 214413 (2003).

34 H. Katsura, I. Maruyama, A. Tanaka, and H. Tasaki, arXiv:0907.4564 1.

35 G. C. Lau, B. G. Ueland, R. S. Freitas, M. L. Dahlberg, P. Schiffer, and R. J. Cava, Phys. Rev. B 73, 012413 (2006).

${ }^{36}$ H. N. Kono and Y. Kuramoto, J. Phys. Soc. Jpn. 75, 084706 (2006).

37 A. Honecker and J. Richter, J. Magn. Magn. Mater. 310, 1331 (2007); A. Honecker, O. Derzhko, and J. Richter, Physica B 404, 3316 (2009).

${ }^{38}$ K. Penc, H. Shiba, F. Mila, and T. Tsukagoshi, Phys. Rev. B 54, 4056 (1996).

39 H. Sakamoto and K. Kubo, J. Phys. Soc. Jpn. 65, 3732
(1996).

40 Ch. Waldtmann, H. Kreutzmann, U. Schollwöck, K. Maisinger, and H.-U. Everts, Phys. Rev. B 62, 9472 (2000).

41 G. B. Martins and W. Brenig, J. Phys.: Condens. Matter 20, 415204 (2008).

42 P. Azaria, C. Hooley, P. Lecheminant, C. Lhuillier, and A. M. Tsvelik, Phys. Rev. Lett. 81, 1694 (1998); Phys. Rev. Lett. 85, 3331 (2000).

43 S. K. Pati and R. R. P. Singh, Phys. Rev. B 60, 7695 (1999).

44 S. R. White and R. R. P. Singh, Phys. Rev. Lett. 85, 3330 (2000).

45 M. Indergand, A. Läuchli, S. Capponi, and M. Sigrist, Phys. Rev. B 74, 064429 (2006).

46 A. Donkov and A. V. Chubukov, Europhys. Lett. 80, 67005 (2007).

47 J. Vidal, R. Mosseri, and B. Douçot, Phys. Rev. Lett. 81, 5888 (1998).

48 H.-J. Schmidt, J. Richter, and R. Moessner, J. Phys. A 39, 10673 (2006).

49 R. J. Baxter, Exactly Solved Models in Statistical Mechanics (Academic Press, London, 1982).

50 Interestingly, for finite (large) $\mathcal{N}$, however, $\xi_{+}$is the largest one among $\xi_{i}$ (when $i= \pm, 3$ or $i= \pm, 3,4,5,6$ ) only if $z$ does not exceed some (large) value $z^{\star}$. Therefore, for fixed $\mu_{0}-\mu>0$ if $T$ becomes smaller than some (small) $T^{\star}=\left(\mu_{0}-\mu\right) / \ln z^{\star}$ a crossover takes place which may produce some tiny features of finite(large)- $\mathcal{N}$ low-temperature thermodynamic quantities.

51 C. Lanczos, J. Res. Nat. Bur. Standards 45, 255 (1950).

52 J. K. Cullum and R. A. Willoughby, Lanczos Algorithms for Large Symmetric Eigenvalue Computations. Vol. I: Theory, SIAM Classics in Applied Mathematics 41 (2002).

53 Y. Nagaoka, Phys. Rev. 147, 392 (1966).

54 M. Yamada and M. Takahashi, J. Phys. Soc. Jpn. 55, 2024 (1986).

55 M. Härtel, J. Richter, D. Ihle, and S.-L. Drechsler, Phys. Rev. B 78, 174412 (2008).

56 Another choice is

$$
\mathbf{T}=\left(\begin{array}{ccc}
1 & \sqrt{z} & \sqrt{z} \\
\sqrt{z} & z & z \\
\sqrt{z} & 0 & z
\end{array}\right)
$$

This matrix has the same eigenvalues as the one in Eq. (A1). 